\title{
New physics models of direct $C P$ violation in charm decays
}

\author{
Wolfgang Altmannshofer, ${ }^{a}$ Reinard Primulando, ${ }^{a, b}$ Chiu-Tien $\mathbf{Y u}^{a, c}$ and Felix $\mathbf{Y u}^{a}$ \\ ${ }^{a}$ Fermi National Accelerator Laboratory, \\ P.O. Box 500, Batavia, IL 60510, U.S.A. \\ ${ }^{b}$ Department of Physics, College of William and Mary, \\ Williamsburg, VA 23187, U.S.A. \\ ${ }^{c}$ Department of Physics, University of Wisconsin, \\ Madison, WI 53706, U.S.A. \\ E-mail: waltmann@fnal.gov, rprimulando@email.wm.edu, cyu27@wisc.edu, \\ felixyu@fnal.gov
}

ABSTRACT: In view of the recent LHCb measurement of $\Delta A_{\mathrm{CP}}$, the difference between the time-integrated $\mathrm{CP}$ asymmetries in $D \rightarrow K^{+} K^{-}$and $D \rightarrow \pi^{+} \pi^{-}$decays, we perform a comparative study of the possible impact of New Physics degrees of freedom on the direct $\mathrm{CP}$ asymmetries in singly Cabibbo suppressed $D$ meson decays. We systematically discuss scenarios with a minimal set of new degrees of freedom that have renormalizable couplings to the SM particles and that are heavy enough such that their effects on the $D$ meson decays can be described by local operators. We take into account both constraints from low energy flavor observables, in particular $D^{0}-\bar{D}^{0}$ mixing, and from direct searches. While models that explain the large measured value for $\Delta A_{\mathrm{CP}}$ with chirally enhanced chromomagnetic penguins are least constrained, we identify a few viable models that contribute to the $D$ meson decays at tree level or through loop induced QCD penguins. We emphasize that such models motivate direct searches at the LHC.

KEYwords: Beyond Standard Model, CP violation

ARXIV EPRINT: 1202.2866 


\section{Contents}

1 Introduction 2

2 CP asymmetries in neutral D meson decays 4

3 Effective Hamiltonian approach $\quad 6$

$3.1 \Delta F=1$ effective Hamiltonian $\quad 6$

$3.2 \Delta F=2$ effective Hamiltonian 8

4 New physics contributions at tree level $\quad 9$

4.1 Flavor changing $Z \quad 9$

$\begin{array}{lll}4.2 & \text { Flavor changing } Z^{\prime} & 11\end{array}$

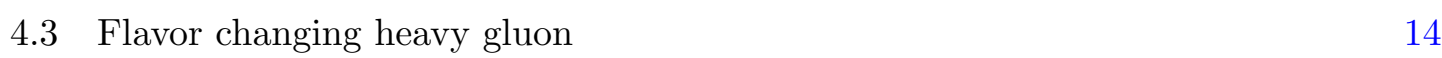

$\begin{array}{ll}4.4 \text { Charged vector boson } & 16\end{array}$

4.5 Two Higgs doublet model 16

$\begin{array}{lll}4.6 & \text { Scalar octet } & 21\end{array}$

$\begin{array}{lll}4.7 & \text { Scalar diquarks } & 25\end{array}$

5 New physics contributions to gluon penguins $\quad 27$

5.1 Fermion + scalar loop without GIM mechanism 27

5.1.1 Dirac fermion 28

$\begin{array}{lll}5.1 .2 & \text { Majorana fermion } & 29\end{array}$

$\begin{array}{lll}5.2 & \text { Fermion + scalar loop with GIM mechanism } 30\end{array}$

5.2.1 Dirac fermion 31

5.2.2 Majorana fermion 32

$\begin{array}{lll}5.3 & \text { Chirally enhanced magnetic penguins } & 32\end{array}$

6 Conclusions $\quad 34$

A Hadronic matrix elements in naïve factorization 36

$\begin{array}{ll}\text { B Anomalous dimensions } & 36\end{array}$

$\begin{array}{ll}\text { C Loop functions } & 37\end{array}$ 


\section{Introduction}

Recently, the LHCb collaboration presented the first evidence for CP violation (CPV) in charm quark decays [1]. In particular, a difference between the time-integrated CP asymmetries in $D \rightarrow K^{+} K^{-}$and $D \rightarrow \pi^{+} \pi^{-}$

$$
\begin{aligned}
\Delta A_{\mathrm{CP}, \mathrm{LHCb}} & =A_{\mathrm{CP}}\left(K^{+} K^{-}\right)-A_{\mathrm{CP}}\left(\pi^{+} \pi^{-}\right) \\
& =(-0.82 \pm 0.21 \pm 0.11) \%
\end{aligned}
$$

has been reported, which is non-zero at $3.5 \sigma$. This measurement is consistent at about the $1 \sigma$ level with the previous measurement from CDF [2], and the previous world average from the Heavy Flavor Averaging Group [3]. The new world average, combining the LHCb result with previous measurements of $A_{\mathrm{CP}}\left(K^{+} K^{-}\right)$and $A_{\mathrm{CP}}\left(\pi^{+} \pi^{-}\right)$at BaBar [4], Belle [5] and $\mathrm{CDF}[2]$, is [3]

$$
\Delta A_{\mathrm{CP}, \text { World Average }}=(-0.645 \pm 0.180) \% \text {. }
$$

The interpretation of this measurement as a sign of New Physics (NP) requires a wellunderstood Standard Model (SM) calculation of this observable. Simple arguments dictate that the SM contribution to direct CPV in $D^{0}$ decays must be both CKM suppressed and loop suppressed. Concretely, the tree level decays $D \rightarrow K^{+} K^{-}$and $D \rightarrow \pi^{+} \pi^{-}$(we implicitly include both $D^{0}$ and $\bar{D}^{0}$ when discussing neutral $D$ decay modes) only involve the first two quark generations, which cannot access the CP violating Kobayashi-Maskawa (KM) phase. The KM phase does enter into the loop-induced gluon penguin diagram for singly-Cabibbo suppressed $D^{0}$ decays that thus can provide both the required weak and strong phase difference relative to the leading SM tree amplitude. This implies that the SM prediction is loop suppressed as well as CKM suppressed, and the naïve expectation for direct $\mathrm{CPV}$ in singly-Cabibbo suppressed $D^{0}$ decays is parametrically given as $\mathcal{O}\left(\left(\alpha_{s} / \pi\right)\left(V_{u b} V_{c b}^{*}\right) /\left(V_{u s} V_{c s}^{*}\right)\right) \sim 10^{-4}$. This leads to the conclusion that the LHCb evidence of CPV at about the percent level is a sign of New Physics.

A precise SM calculation, however, is difficult to accomplish. Although tree level and loop level SM contributions to the quark level processes $c \rightarrow u s \bar{s}$ and $c \rightarrow u d \bar{d}$ are readily calculated, the evaluation of the hadronic matrix elements $\left\langle K^{+} K^{-}\left|\left(\bar{u} \Gamma_{1} s\right)\left(\bar{s} \Gamma_{2} c\right)\right| D^{0}\right\rangle$, for example, is not easily performed. In the simplest approach, naïve factorization, the hadronic matrix elements are "factorized" into $\left\langle K^{+}\right|\left(\bar{u} \Gamma_{1} s|0\rangle\left\langle K^{-}\left|\left(\bar{s} \Gamma_{2} c\right)\right| D^{0}\right\rangle\right.$ which is formally the leading term in the heavy charm quark limit. As the charm mass is close to $\Lambda_{\mathrm{QCD}}$, however, this approach suffers from large $1 / m_{c}$ power corrections. In particular, so-called annihilation diagrams are ignored, where quarks are pair-produced from the vacuum to complete the $K$ or $\pi$ mesons, as are long-range QCD effects such as final state rescattering, where constituent $s$ quarks of a $D \rightarrow K^{+} K^{-}$decay rescatter into $d$ quarks of a $\pi^{+} \pi^{-}$final state. Alternative techniques such as the topological diagram approach organize decay and annihilation amplitudes according to weak current insertions and $\mathrm{SU}(3)_{F}$ light quark flavor symmetry, and then try to extract amplitudes and phases directly from $D^{0}$ branching ratio data.

Several recent papers have dicussed improved estimates for $\Delta A_{\mathrm{CP}}$ in the SM. In [6], a NLO QCD factorization calculation is amended by an estimate of the effect of certain 
$1 / m_{c}$ suppressed penguin amplitudes using $D^{0}$ branching ratio data. Assuming an $\mathcal{O}(1)$ strong phase, the authors find the SM can potentially give $\left|\Delta A_{\mathrm{CP}}\right| \sim 0.4 \%$. As their result admittedly neglects several effects which could alternatively reduce or enhance this estimate, they conclude that the measured value of $\Delta A_{\mathrm{CP}}$ could be reproduced in the SM. Studies that inform magnitudes and phases of $D^{0}$ meson decay amplitudes directly from data were performed in [7-9] following a topological diagram approach. The authors of [7] arrive at a slightly smaller $\Delta A_{\mathrm{CP}} \sim-0.25 \%$ estimate, which remains, they highlight, more than $2 \sigma$ away from the world average. In $[8,9]$, the correlation between direct CPV in $D \rightarrow K^{+} K^{-}$and $\pi^{+} \pi^{-}$and other $D$ meson decays is emphasized as an important cross-check of the $\mathrm{LHCb}$ result.

Even though there is large uncertainty in the SM value of $\Delta A_{\mathrm{CP}}$, it is nevertheless important and exciting to consider the possibility that we are seeing evidence of NP. Literature prior to the $\mathrm{LHCb}$ result emphasized the continued fact that $\mathrm{CPV}$ in the charm sector is considered an excellent probe of NP beyond the SM [10-12]. Among the most promising probes of CPV in the charm sector are observables in $D^{0}-\bar{D}^{0}$ mixing [13-17] and singly Cabibbo suppressed $D$ decays [18-20]. In fact, since flavor physics observables can probe energy scales much higher than those directly measured, we could potentially expect that NP at the LHC would first be seen from its flavor effects at low energies and only later accessed directly.

Our goal is to investigate the possibility that NP is indeed responsible for the large $\Delta A_{\mathrm{CP}}$ measurement and to outline the corresponding NP parameter space consistent with all experimental constraints for a variety of NP models. Some recent work has discussed the NP possibility both model independently [21] and in the context of various concrete NP scenarios, including up-type flavor changing neutral currents (FCNCs), fourth-generation fermions, $R$-parity violating supersymmetry, and the MSSM with nonstandard sources of flavor violation $[22-26]$.

Our work differs from these previous analyses since we consider a much broader range of new NP possibilities and apply a full gamut of experimental constraints, both from low energy experiments and collider searches. In particular, we systematically discuss models with a minimal set of new degrees of freedom with renormalizable couplings to the SM particles and are heavy enough such that their effects on the $D$ meson decays can be described by local operators. Specifically, we consider models with new massive neutral gauge bosons that have flavor changing tree level couplings to quarks, models with extended scalar sectors, and models where the $D \rightarrow K^{+} K^{-}$and $D \rightarrow \pi^{+} \pi^{-}$decays are modified at the loop level by gluon penguins. As discussed in [18, 21, 26], the loop induced $\Delta F=1$ chromomagnetic dipole operator (here and throughout, $F$ refers to charm number except where noted) is, on general grounds, expected to be the least constrained approach for generating large nonstandard effects in $D^{0}$ meson decays. On the other hand, the effects of four fermion operators that are, for example, induced by tree level exchange of flavor changing NP degrees of freedom, are highly constrained by $D^{0}-\bar{D}^{0}$ mixing data. As is well known, the $D^{0}-\bar{D}^{0}$ constraints become more effective with heavier NP degrees of freedom [18], leading to the expectation that almost no NP parameter space remains in models where four fermion operators are responsible for nonstandard direct CPV in 
$D \rightarrow K^{+} K^{-}$and $D \rightarrow \pi^{+} \pi^{-}$. Our work shows to what extent this naïve expectation holds true and identifies a few exceptions. We emphasize that each of the viable NP possibilities that can accomodate a large $\Delta A_{\mathrm{CP}}$ motivates a further phenomenological study focusing on the allowed parameter space identified in this work, which we leave for a future study.

In section 2, we review aspects of CPV in neutral $D$ meson decays that are most relevant for our analysis. In section 3 , we present the $\Delta F=1$ and $\Delta F=2$ effective Hamiltonians that can describe NP contributions to the $D \rightarrow K^{+} K^{-}$and $D \rightarrow \pi^{+} \pi^{-}$ decays and to $D^{0}-\bar{D}^{0}$ mixing, respectively. The various NP models that contribute to CPV at tree level are discussed in section 4, while the NP models that contribute at loop level are discussed in section 5 . We conclude in section 6 . Technical details about hadronic matrix elements and renormalization group running, as well as a collection of loop functions can be found in the appendices.

\section{$2 \mathrm{CP}$ asymmetries in neutral $\mathrm{D}$ meson decays}

The neutral $D$ meson mass eigenstates $D_{1}$ and $D_{2}$ are linear combinations of the strong interaction eigenstates, $D^{0}$ and $\bar{D}^{0}$

$$
\left|D_{1,2}\right\rangle=p\left|D^{0}\right\rangle \pm q\left|\bar{D}^{0}\right\rangle
$$

The factors $q$ and $p$ are given by

$$
\frac{q}{p}=\sqrt{\frac{M_{12}^{*}-\frac{i}{2} \Gamma_{12}^{*}}{M_{12}-\frac{i}{2} \Gamma_{12}}},
$$

where $M_{12}$ and $\Gamma_{12}$ are the dispersive and absorptive part of the $D$ meson mixing amplitude. CP violation in $D$ meson mixing is signaled by $|q / p| \neq 1$ or $\phi=\operatorname{Arg}(q / p) \neq 0$.

The normalized mass and width differences, $x$ and $y$, in the neutral $D$ meson system are given by

$$
\begin{aligned}
& x=\frac{\Delta M_{D}}{\Gamma}=2 \tau \operatorname{Re}\left[\frac{q}{p}\left(M_{12}-\frac{i}{2} \Gamma_{12}\right)\right], \\
& y=\frac{\Delta \Gamma_{D}}{2 \Gamma}=-2 \tau \operatorname{Im}\left[\frac{q}{p}\left(M_{12}-\frac{i}{2} \Gamma_{12}\right)\right],
\end{aligned}
$$

where the lifetime of the $D^{0}$ mesons $\tau=1 / \Gamma=0.41 \mathrm{ps}[10]$.

The time integrated $\mathrm{CP}$ asymmetry in the decay of neutral $D$ mesons to a final CP eigenstate $f=K^{+} K^{-}, \pi^{+} \pi^{-}$is defined as

$$
\begin{aligned}
A_{\mathrm{CP}}(f) & =\frac{\Gamma\left(D^{0} \rightarrow f\right)-\Gamma\left(\bar{D}^{0} \rightarrow f\right)}{\Gamma\left(D^{0} \rightarrow f\right)+\Gamma\left(\bar{D}^{0} \rightarrow f\right)} \\
& =A^{m}+A^{i}+A_{f}^{d} .
\end{aligned}
$$

The time integrated $\mathrm{CP}$ asymmetry receives contributions from CPV in mixing $A^{m}$, CPV in interference of decays with and without mixing $A^{i}$, and from CPV in the decay itself 
$A_{f}^{d}$. The "indirect" $\mathrm{CP}$ asymmetries $A^{m}$ and $A^{i}$ are approximately independent of the final state and depend only on $D^{0}-\bar{D}^{0}$ mixing parameters

$$
\begin{aligned}
A^{m} & =\eta_{\mathrm{CP}}^{f} \frac{y}{2}\left(\left|\frac{p}{q}\right|-\left|\frac{q}{p}\right|\right) \cos \phi, \\
A^{i} & =\eta_{\mathrm{CP}}^{f} \frac{x}{2}\left(\left|\frac{p}{q}\right|+\left|\frac{q}{p}\right|\right) \sin \phi,
\end{aligned}
$$

where $\eta_{\mathrm{CP}}^{f}$ is the CP parity of the final state. The "direct" CP asymmetry $A_{f}^{d}$ is instead sensitive to the final state. The decay amplitudes of singly Cabibbo suppressed $D$ meson decays $A\left(D^{0} \rightarrow f\right)=A_{f}$ and $A\left(\bar{D}^{0} \rightarrow f\right)=\bar{A}_{f}$ can be written as [18]

$$
\begin{aligned}
& A_{f}=A_{f}^{T}\left(1+r_{f} e^{i\left(\delta_{f}+\phi_{f}\right)}\right), \\
& \bar{A}_{f}=\eta_{\mathrm{CP}}^{f} A_{f}^{T}\left(1+r_{f} e^{i\left(\delta_{f}-\phi_{f}\right)}\right),
\end{aligned}
$$

where $A_{f}^{T}$ is the dominant singly Cabibbo suppressed tree level amplitude, which can be taken real by convention, and $r_{f}$ is the relative size of subleading ("penguin") amplitudes. With respect to the tree amplitude, the penguin amplitudes can have a relative weak phase $\phi_{f}$ and a relative strong phase $\delta_{f}$.

Under the assumption that $r_{f}$ is small, one arrives at the following expression for the direct $\mathrm{CP}$ asymmetry

$$
A_{f}^{d}=2 r_{f} \sin \delta_{f} \sin \phi_{f} .
$$

The difference between the time-integrated $\mathrm{CP}$ asymmetries in $D \rightarrow K^{+} K^{-}$and $D \rightarrow$ $\pi^{+} \pi^{-}$measured by LHCb is given by [1]

$$
\Delta A_{\mathrm{CP}}=A_{K^{+} K^{-}}^{d}-A_{\pi^{+} \pi^{-}}^{d}+\frac{\Delta\langle t\rangle}{\tau}\left(A^{m}+A^{i}\right),
$$

where $\Delta\langle t\rangle / \tau=(9.8 \pm 0.9) \%$ is a small difference in the average decay times of the $D^{0}$ mesons in the $K^{+} K^{-}$and $\pi^{+} \pi^{-}$sample [1]. Given the existing bounds on the indirect $\mathrm{CP}$ asymmetries [3], the $\mathrm{LHCb}$ measurement of $\Delta A_{\mathrm{CP}}$ is an excellent approximation of the difference in the direct $\mathrm{CP}$ asymmetries.

As already mentioned in the Introduction, charm CPV in the SM is strongly Cabibbo suppressed. Furthermore, in the SM, direct CP violation in $D \rightarrow K^{+} K^{-}$and $D \rightarrow \pi^{+} \pi^{-}$ decays comes from the interference of the tree level contribution with a loop suppressed penguin amplitude and correspondingly, $r_{f} \sim \mathcal{O}\left(\alpha_{s} / \pi\right)\left(V_{u b} V_{c b}^{*}\right) /\left(V_{u s} V_{c s}^{*}\right) \sim 10^{-4}$. Even though the weak phase of the SM penguin is large $\left(\gamma \sim 70^{\circ}\right)$ and assuming a maximal strong phase, a naïve SM estimate for $\Delta A_{\mathrm{CP}}$ is therefore smaller than the global average by at least an order of magnitude.

Sizable direct $\mathrm{CP}$ asymmetries in the $D \rightarrow K^{+} K^{-}$and $D \rightarrow \pi^{+} \pi^{-}$decays are only possible in the SM if the relevant hadronic matrix elements are strongly enhanced [27]. Despite several recent studies [6-8], it remains unclear to what extent such an enhancement is present and whether the value of $\Delta A_{\mathrm{CP}}$ measured by LHCb can be explained within the SM.

In the following we investigate the possibility that the measured $\Delta A_{\mathrm{CP}}$ is due to New Physics. 


\section{$3 \quad$ Effective Hamiltonian approach}

\section{1 $\Delta F=1$ effective Hamiltonian}

In the New Physics frameworks discussed below, contributions to the singly Cabibbo suppressed $D \rightarrow K^{+} K^{-}$and $D \rightarrow \pi^{+} \pi^{-}$decays can be described by the following effective Hamiltonian

$$
\begin{aligned}
\mathcal{H}_{\mathrm{eff}}= & \left(\sum_{p} \lambda_{p} \sum_{i=1}^{2}\left(C_{i}^{(1) p} O_{i}^{(1) p}+\tilde{C}_{i}^{(1) p} \tilde{O}_{i}^{(1) p}\right)\right. \\
& \left.+\sum_{i}\left(C_{i}^{(1)} O_{i}^{(1)}+\tilde{C}_{i}^{(1)} \tilde{O}_{i}^{(1)}\right)\right)+ \text { h.c. }
\end{aligned}
$$

where $\lambda_{p}=V_{c p} V_{u p}^{*}$, and the operators $O_{i}^{(1)}$ are given by

$$
\begin{aligned}
O_{1}^{(1) p} & =(\bar{u} p)_{V-A}(\bar{p} c)_{V-A}, \\
O_{2}^{(1) p} & =\left(\bar{u}_{\alpha} p_{\beta}\right)_{V-A}\left(\bar{p}_{\beta} c_{\alpha}\right)_{V-A}, \\
O_{3}^{(1)} & =(\bar{u} c)_{V-A} \sum_{q}(\bar{q} q)_{V-A}, \\
O_{4}^{(1)} & =\left(\bar{u}_{\alpha} c_{\beta}\right)_{V-A} \sum_{q}\left(\bar{q}_{\beta} q_{\alpha}\right)_{V-A}, \\
O_{5}^{(1)} & =(\bar{u} c)_{V-A} \sum_{q}(\bar{q} q)_{V+A}, \\
O_{6}^{(1)} & =\left(\bar{u}_{\alpha} c_{\beta}\right)_{V-A} \sum_{q}\left(\bar{q}_{\beta} q_{\alpha}\right)_{V+A}, \\
O_{7}^{(1)} & =\frac{3}{2}(\bar{u} c)_{V-A} \sum_{q} e_{q}(\bar{q} q)_{V+A}, \\
O_{8}^{(1)} & =\frac{3}{2}\left(\bar{u}_{\alpha} c_{\beta}\right)_{V-A} \sum_{q} e_{q}\left(\bar{q}_{\beta} q_{\alpha}\right)_{V+A}, \\
O_{9}^{(1)} & =\frac{3}{2}(\bar{u} c)_{V-A} \sum_{q} e_{q}(\bar{q} q)_{V-A}, \\
O_{10}^{(1)} & =\frac{3}{2}\left(\bar{u}_{\alpha} c_{\beta}\right)_{V-A} \sum_{q} e_{q}\left(\bar{q}_{\beta} q_{\alpha}\right)_{V-A}, \\
O_{8 g}^{(1)} & =\frac{g_{s}}{8 \pi^{2}} m_{c} \bar{u} \sigma^{\mu \nu}\left(1+\gamma_{5}\right) c_{\beta} t_{\alpha \beta}^{a} G_{\mu \nu}^{a}, \\
O_{S 1}^{(1)} & =\left(\bar{u} P_{L} s\right)\left(\bar{s} P_{L} c\right), \\
O_{S 2}^{(1)} & =\left(\bar{u}_{\alpha} P_{L} s_{\beta}\right)\left(\bar{s}_{\beta} P_{L} c_{\alpha}\right), \\
O_{T 1}^{(1)} & =\left(\bar{u} \sigma_{\mu \nu} P_{L} s\right)\left(\bar{s} \sigma^{\mu \nu} P_{L} c\right), \\
O_{T 2}^{(1)} & =\left(\bar{u}_{\alpha} \sigma_{\mu \nu} P_{L} s_{\beta}\right)\left(\bar{s}_{\beta} \sigma^{\mu \nu} P_{L} c_{\alpha}\right) .
\end{aligned}
$$

The index $q$ runs over all active quark flavors, the index $p$ runs over all active down type quark flavors, $\alpha$ and $\beta$ are color indices (that are implicitly summed over), $e_{q}$ is the electric 
charge of the quark $q,(V \pm A)$ refers to the Dirac structures $\gamma_{\mu}\left(1 \pm \gamma_{5}\right), P_{R, L}=\frac{1}{2}\left(1 \pm \gamma_{5}\right)$ and $\sigma_{\mu \nu}=\frac{i}{2}\left(\gamma_{\mu} \gamma_{\nu}-\gamma_{\nu} \gamma_{\mu}\right)$. The operators $\tilde{O}_{i}^{(1)\{p\}}$ are obtained from $O_{i}^{(1)\{p\}}$ by replacing $\gamma_{5} \rightarrow-\gamma_{5}$.

The operators $O_{1,2}^{(1) p}$ are the so-called current-current operators. In the SM, tree level $W$ exchange generates at the matching scale the Wilson coefficient $C_{1}^{(1) p} \simeq G_{F} / \sqrt{2}$. The QCD penguin operators $O_{3,4,5,6}^{(1)}$ and the chromomagnetic operator $O_{8 g}^{(1)}$ are first generated at $\mathcal{O}\left(\alpha_{s}\right)$ and proportional to $V_{u b} V_{c b}^{*}$. The chromomagnetic operator is proportional to the charm quark mass but can be chirally enhanced by $v / m_{c}$ from NP. The QED penguin operators $O_{7,8,9,10}^{(1)}$ are also proportional to $V_{u b} V_{c b}^{*}$. They are of $\mathcal{O}(\alpha)$ and negligible in the $D \rightarrow K^{+} K^{-}$and $D \rightarrow \pi^{+} \pi^{-}$decays in the SM. In the NP models discussed below that have tree level contributions to the $D \rightarrow K^{+} K^{-}$and $D \rightarrow \pi^{+} \pi^{-}$decay amplitudes, however, the QED penguin operators can be relevant. The scalar operators $O_{S 1, S 2}^{(1)}$ become important in the context of the $2 \mathrm{HDM}$ discussed in section 4.5 and the scalar octet discussed in section 4.6. The tensor operators $O_{T 1, T 2}^{(1)}$ do not contribute to $D \rightarrow K^{+} K^{-}$and $D \rightarrow$ $\pi^{+} \pi^{-}$decays in naïve factorization. We consider them nonetheless, because they mix with the scalar operators under renormalization group running.

The ratio $r_{f}$ that enters the expression for the direct CP asymmetry eq. (2.9) can be written as a function of the Wilson coefficients appearing in eq. (3.1). We use the results from [18] for the hadronic matrix elements that are obtained using naïve factorization for $O_{1, \ldots, 6}^{(1)}$ and QCD factorization $[28,29]$ for $O_{8 g}^{(1)}$. The matrix elements obtained in naïve factorization are formally the leading terms in an expansion in $\alpha_{s}$ and $\Lambda_{\mathrm{QCD}} / m_{c}[28,29]$. In the case of $D$ meson decays, however, and as mentioned in the Introduction, it is known that power corrections, in particular annihilation contributions, which are formally suppressed by $1 / m_{c}$, can be equally important $[6,8,18]$. The naïve factorization results can therefore only be considered as rough estimates and in our numerical analysis, we will allow for enhancements up to a plausible factor of $3[6,18]$. For our analysis, we extend the results for the hadronic matrix elements given in [18] by including the QED penguin and scalar operators (see appendix A for details). We find

$$
\begin{aligned}
r_{f} e^{i \phi_{f}} \simeq & \frac{1}{\lambda_{p}}\left(C_{1}^{(1) p}+\frac{C_{2}^{(1) p}}{N_{c}}\right)^{-1}\left(\frac{\lambda_{p}\left(C_{2}^{(1) p}\right)_{\mathrm{NP}}}{N_{c}}+C_{4}^{(1)}+\frac{C_{3}^{(1)}}{N_{c}}-\frac{C_{10}^{(1)}}{2}-\frac{C_{9}^{(1)}}{2 N_{c}}-\frac{3 \alpha_{s}}{4 \pi} \frac{N_{c}^{2}-1}{N_{c}^{2}} C_{8 g}^{(1)}\right. \\
& \left.+\chi_{f}\left(C_{6}^{(1)}+\frac{C_{5}^{(1)}}{N_{c}}-\frac{C_{8}^{(1)}}{2}-\frac{C_{7}^{(1)}}{2 N_{c}}-\frac{C_{S 1}^{(1)}}{8}-\frac{C_{S 2}^{(1)}}{8 N_{c}}-\frac{\alpha_{s}}{4 \pi} \frac{N_{c}^{2}-1}{N_{c}^{2}} C_{8 g}^{(1)}\right)+\left(C_{i}^{(1)} \leftrightarrow \tilde{C}_{i}^{(1)}\right)\right),
\end{aligned}
$$

where $N_{c}=3$ is the number of colors and $p=s, f=K^{+} K^{-}$for the $D \rightarrow K^{+} K^{-}$decay and $p=d, f=\pi^{+} \pi^{-}$for the $D \rightarrow \pi^{+} \pi^{-}$decay. The chiral factors $\chi_{f}$ are approximately given by

$$
\chi_{K^{+} K^{-}} \simeq \frac{2 m_{K}^{2}}{m_{c} m_{s}}, \quad \chi_{\pi^{+} \pi^{-}} \simeq \frac{2 m_{\pi}^{2}}{m_{c}\left(m_{d}+m_{u}\right)},
$$

with all quark masses evaluated at the scale of the $D$ meson $\mu \simeq m_{D} \simeq 1.8 \mathrm{GeV}$. All the Wilson coefficients in eq. (3.3) are evaluated at this scale. We use LO renormalization group 
running for $C_{1, \ldots, 10}^{(1)}$ and $C_{S 1, S 2, T 1, T 2}^{(1)}$ as well as for $C_{8 g}^{(1)}$ to evolve the Wilson coefficients from the high matching scale, where NP degrees of freedom are integrated out, down to $\mu \simeq m_{D}$. The corresponding anomalous dimensions are collected in appendix B. We do not include 2-loop mixing between $C_{8 g}^{(1)}$ and the other Wilson coefficients. In view of the large uncertainties in the evaluation of the hadronic matrix elements, we consider this approximate approach to be fully justified.

While there are no strong phase differences between the several operator matrix elements in the naïve factorization approach, they can be generated by large power corrections or final state interactions. Throughout this work, we will assume $\mathcal{O}(1)$ strong phase differences, following $[6,18]$.

\section{2 $\Delta F=2$ effective Hamiltonian}

In the models discussed below, the most important flavor constraints come often from $D^{0}-\bar{D}^{0}$ and $K-\bar{K}$ mixing. New Physics contributions to meson mixing can be described by the effective Hamiltonian

$$
\mathcal{H}_{\text {eff }}=\sum_{i=1}^{5} C_{i}^{(2)} O_{i}^{(2)}+\sum_{i=1}^{3} \tilde{C}_{i}^{(2)} \tilde{O}_{i}^{(2)}+\text { h.c. } .
$$

In the case of $D^{0}-\bar{D}^{0}$ mixing, the most important operators for our analysis are given by

$$
\begin{aligned}
& O_{1}^{(2) D}=\left(\bar{u}_{\alpha} \gamma_{\mu} P_{L} c_{\alpha}\right)\left(\bar{u}_{\beta} \gamma^{\mu} P_{L} c_{\beta}\right), \\
& \tilde{O}_{1}^{(2) D}=\left(\bar{u}_{\alpha} \gamma_{\mu} P_{R} c_{\alpha}\right)\left(\bar{u}_{\beta} \gamma^{\mu} P_{R} c_{\beta}\right), \\
& \tilde{O}_{2}^{(2) D}=\left(\bar{u}_{\alpha} P_{R} c_{\alpha}\right)\left(\bar{u}_{\beta} P_{R} c_{\beta}\right) .
\end{aligned}
$$

In the case of $K-\bar{K}$ mixing, the operators most relevant for our analysis are

$$
\begin{aligned}
O_{1}^{(2) K} & =\left(\bar{d}_{\alpha} \gamma_{\mu} P_{L} s_{\alpha}\right)\left(\bar{d}_{\beta} \gamma^{\mu} P_{L} s_{\beta}\right), \\
\tilde{O}_{1}^{(2) K} & =\left(\bar{d}_{\alpha} \gamma_{\mu} P_{R} s_{\alpha}\right)\left(\bar{d}_{\beta} \gamma^{\mu} P_{R} s_{\beta}\right), \\
O_{4}^{(2) K} & =\left(\bar{d}_{\alpha} P_{L} s_{\alpha}\right)\left(\bar{d}_{\beta} P_{R} s_{\beta}\right), \\
O_{5}^{(2) K} & =\left(\bar{d}_{\alpha} P_{L} s_{\beta}\right)\left(\bar{d}_{\beta} P_{R} s_{\alpha}\right) .
\end{aligned}
$$

In the above expressions, $P_{R, L}=\frac{1}{2}\left(1 \pm \gamma_{5}\right)$ and $\alpha, \beta$ are color indices (that are implicitly summed over).

The Wilson coefficients $C_{i}^{(2)}$ are again obtained by integrating out the NP degrees of freedom at a scale of the order of the mass of the new particles. Using renormalization group evolution [30,31], these coefficients are subsequently run down to the low scale where the hadronic matrix elements [32-34] are given. Combining Wilson coefficients with the hadronic matrix elements gives the NP contribution to the dispersive part of the mixing amplitude $M_{12} \cdot{ }^{1}$ In the case of $D^{0}-\bar{D}^{0}$ mixing, the SM contributions to neither the dispersive part nor the absorptive part of the mixing amplitude can be predicted reliably as they are dominated by long distance effects [35, 36]. In our numerical analysis, we

\footnotetext{
${ }^{1}$ The absorptive part of the mixing amplitude $\Gamma_{12}$ is not sensitive to new short distance dynamics.
} 
allow the long distance contributions to vary in the ranges $M_{12}^{\mathrm{LD}} \in[-0.02,0.02] \mathrm{ps}^{-1}$ and $\Gamma_{12}^{\mathrm{LD}} \in[-0.04,0.04] \mathrm{ps}^{-1}[37]$, such that by themselves they can saturate the experimental values. We apply the most recent averages and $1 \sigma$ errors of the experimental results on the $D^{0}-\bar{D}^{0}$ mixing parameters [3]

$$
\begin{aligned}
x & =\left(0.63_{-0.20}^{+0.19}\right) \%, & y & =(0.75 \pm 0.12) \%, \\
|q / p| & =0.89_{-0.15}^{+0.17}, & \phi & =\left(-10.1_{-8.8}^{+9.4}\right)^{\circ},
\end{aligned}
$$

at the $2 \sigma$ level throughout our analysis.

We note that in many of our minimal field content scenarios, the NP vertices used in the $\Delta F=1$ operators are also used for the $\Delta F=2$ operators, leading to a phase relation $2 \phi_{F=1}=\phi_{F=2}$ between the CPV for $D^{0}$ decays and the CPV for $D^{0}-\bar{D}^{0}$ mixing. This relation implies that the $D^{0}-\bar{D}^{0}$ mixing constraint is best satisfied by eliminating the CPV in $D^{0}-\bar{D}^{0}$ mixing and saturating the $D^{0}-\bar{D}^{0}$ mixing transition amplitude. On the other hand, in non-minimal constructions, this phase relation could be different, possibly making the null observation of $\mathrm{CPV}$ in $D^{0}-\bar{D}^{0}$ mixing the more restrictive constraint.

\section{New physics contributions at tree level}

We concentrate on New Phyiscs models where the new degrees of freedom are heavy enough such that their effects in low energy observables can be reliably described by the local operators introduced in section 3. We do not consider scenarios with very light mediators, which is beyond the scope of this work. Moreover, we focus on models where the new degrees of freedom have renormalizable couplings to SM degrees of freedom.

In this section, we analyze models where at most one new field is added to the SM. We first discuss extensions of the SM in which a massive neutral gauge boson leads to tree level contributions to the $D \rightarrow K^{+} K^{-}$and $D \rightarrow \pi^{+} \pi^{-}$decay ampitudes. We consider a flavor changing coupling of the SM $Z$ boson in section 4.1, a flavor changing $Z^{\prime}$ in section 4.2 and a flavor changing heavy gluon in section 4.3. We also comment on the possible effects of a new charged gauge boson in section 4.4. Then, we analyze models with extended scalar sectors, namely a 2 Higgs doublet model with Minimal Flavor Violation in section 4.5, a model with a scalar octet in section 4.6 and a model with a scalar diquark in section 4.7.

Models that contain more than one non-SM particle and where NP contributions to the $D$ meson decays are first generated at the one loop level are discussed in section 5 .

\subsection{Flavor changing $Z$}

We consider a flavor changing coupling of the SM $Z$ boson to the right-handed charm and up quark

$$
\mathcal{L}_{\text {int }}=X_{c u} \bar{c}_{R} \gamma^{\mu} u_{R} Z_{\mu}+\text { h.c. },
$$

where $X_{c u}$ is a complex parameter. A complementary setup, where flavor changing couplings involving the top quark generate an effective $c \rightarrow u$ transition at the loop level by a double flavor flip $c \rightarrow t \rightarrow u$, is discussed in [26]. Flavor changing $Z$ couplings can appear in various scenarios [38], for example in models with non-sequential generations of quarks [39] 


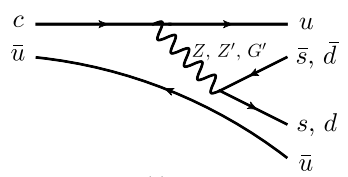

(a)

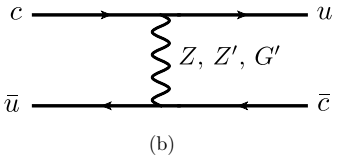

Figure 1. Example tree level Feynman diagrams that contribute (a) to the $D \rightarrow K^{+} K^{-}$and $D \rightarrow \pi^{+} \pi^{-}$decay amplitudes and (b) to $D^{0}-\bar{D}^{0}$ mixing in the cases of a flavor changing $Z, Z^{\prime}$ and heavy gluon $G^{\prime}$.

and also in models with extra U(1) gauge symmetries [40], or can be loop induced as in SUSY models. In the absence of $\mathrm{SU}(2)_{L}$ breaking sources, the $\bar{c}_{R} u_{R} Z$ coupling has the form of a charge radius interaction and vanishes for $q^{2} \rightarrow 0$, where $q$ is the momentum of the $Z$ boson. The dominant contribution to the coupling $X_{c u}$ is therefore in general expected to be proportional to $v^{2} / \Lambda_{\mathrm{NP}}^{2}$, where $v$ is the Higgs vacuum expectation value (vev) and $\Lambda_{\mathrm{NP}}$ is the NP scale where the flavor changing $Z$ coupling is generated.

As shown in diagram (a) of figure 1, the $X_{c u}$ coupling leads to tree level contributions to the Wilson coefficients $\tilde{C}_{5}^{(1)}, \tilde{C}_{7}^{(1)}$ and $\tilde{C}_{9}^{(1)}$

$$
\begin{aligned}
& \tilde{C}_{5}^{(1)}=-\frac{1}{3} \frac{g}{2 c_{W}} \frac{X_{c u}^{*}}{4 M_{Z}^{2}}, \\
& \tilde{C}_{7}^{(1)}=\frac{2}{3} g c_{W} \frac{X_{c u}^{*}}{4 M_{Z}^{2}}, \\
& \tilde{C}_{9}^{(1)}=-\frac{2}{3} \frac{g s_{W}^{2}}{c_{W}} \frac{X_{c u}^{*}}{4 M_{Z}^{2}} .
\end{aligned}
$$

The flavor changing $\bar{c} u Z$ coupling also inevitably generates tree level contributions to $D^{0}-$ $\bar{D}^{0}$ mixing

$$
\tilde{C}_{1}^{(2) D}=\frac{\left(X_{c u}^{*}\right)^{2}}{2 M_{Z}^{2}} .
$$

If the $Z$ boson has flavor changing couplings to left-handed quarks, 1-loop contributions to $\epsilon^{\prime} / \epsilon$ would also be generated. In order to avoid this constraint, we restrict ourselves to the $\bar{c}_{R} u_{R} Z$ coupling.

In figure 2, we show the regions in the $\left|X_{c u}\right|-\operatorname{Arg}\left(X_{c u}\right)$ plane that are compatible with the range for $\Delta A_{\mathrm{CP}}$ in eq. (1.2) at the $1 \sigma$ level. The green (solid) band is obtained using the expressions for the decay amplitude in naïve factorization. The blue (dashed) band assumes an enhancement of the hadronic matrix elements by a factor of 3 . The red (dash-dotted) region is excluded by the constraints from $D^{0}-\bar{D}^{0}$ mixing. The $D^{0}-\bar{D}^{0}$ constraints are minimized for $\operatorname{Arg}\left(X_{c u}\right)=0, \pi / 2, \pi, 3 \pi / 2$, where constraints from CPV in $D^{0}-\bar{D}^{0}$ are not effective and the dominant constraint comes from the normalized mass difference $x$. Indeed, if no enhancement of the hadronic matrix elements is assumed, sizeable NP effects in $\Delta A_{\mathrm{CP}}$ are only compatible with $D^{0}-\bar{D}^{0}$ mixing in a small corner of parameter space with $\operatorname{Arg}\left(X_{c u}\right) \simeq \pi / 2,3 \pi / 2$. Still, barring the finetuned situations 


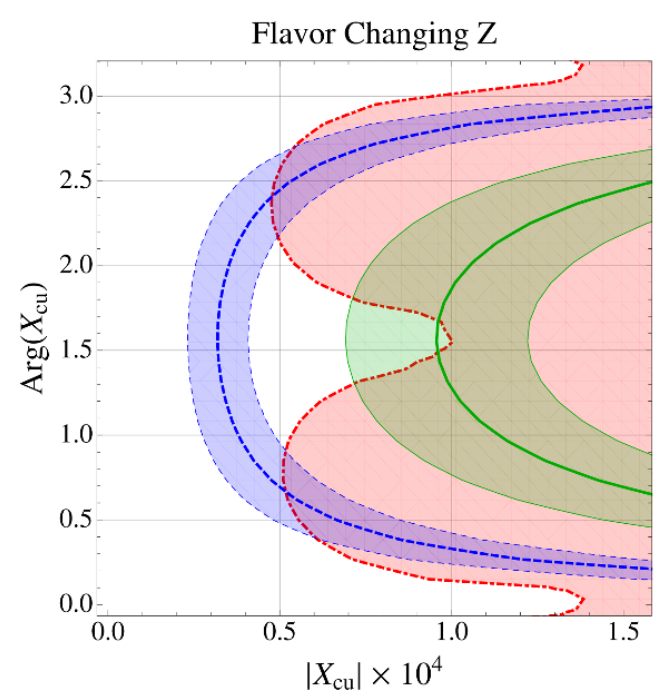

Figure 2. Regions in the $\left|X_{c u}\right|-\operatorname{Arg}\left(X_{c u}\right)$ plane compatible with the data on $\Delta A_{\mathrm{CP}}$, wA at the $1 \sigma$ level in the model with a flavor changing $Z$. The green (solid) band corresponds to the expressions for the decay amplitude in naïve factorization, the blue (dashed) band assumes an enhancement of the hadronic matrix elements by a factor of 3 . The red (dash-dotted) region is excluded by the $D^{0}-\bar{D}^{0}$ mixing constraints.

$\operatorname{Arg}\left(X_{c u}\right)=\pi / 2,3 \pi / 2$, sizeable NP effects in $\Delta A_{\mathrm{CP}}$ also imply indirect CPV in $D^{0}-\bar{D}^{0}$ mixing close to the current experimental bounds. The required size of the flavor changing coupling $v^{2} / \Lambda_{\mathrm{NP}}^{2} \propto\left|X_{c u}\right| \simeq 10^{-4}$ points towards a NP scale of $\Lambda_{\mathrm{NP}} \lesssim$ few $\times 10 \mathrm{TeV}$, where this coupling is generated and not necessarily within the immediate reach of direct searches.

\subsection{Flavor changing $Z^{\prime}$}

Next, we consider a leptophobic massive $Z^{\prime}$ gauge boson with tree level flavor changing couplings to right-handed up and charm quarks. Models of this type can be easily constructed if the $Z^{\prime}$ couples with SM degrees of freedom through higher dimensional operators [41]. Flavor changing couplings of a $Z^{\prime}$ can also arise, for example, in models with family non-universal couplings [40,42]. We parameterize the interactions of the $Z^{\prime}$ in the following way

$$
\begin{aligned}
\mathcal{L}_{\text {int }}= & g_{L} \bar{u}_{L}^{i} \gamma^{\mu} u_{L}^{i} Z_{\mu}^{\prime}+g_{u} \bar{u}_{R}^{i} \gamma^{\mu} u_{R}^{i} Z_{\mu}^{\prime} \\
& +g_{L} \bar{d}_{L}^{i} \gamma^{\mu} d_{L}^{i} Z_{\mu}^{\prime}+g_{d} \bar{d}_{R}^{i} \gamma^{\mu} d_{R}^{i} Z_{\mu}^{\prime} \\
& +X_{c u} \bar{c}_{R} \gamma^{\mu} u_{R} Z_{\mu}^{\prime}+\text { h.c. },
\end{aligned}
$$

where the flavor universal couplings $g_{u}, g_{d}$ and $g_{L}$ are free real parameters and the (small) flavor changing coupling $X_{c u}$ is a free, complex parameter. We restrict ourselves to a tree level $c \rightarrow u$ coupling and do not consider $t \rightarrow c$ and $t \rightarrow u$ couplings that could induce the $c \rightarrow u$ transition at the loop level. 

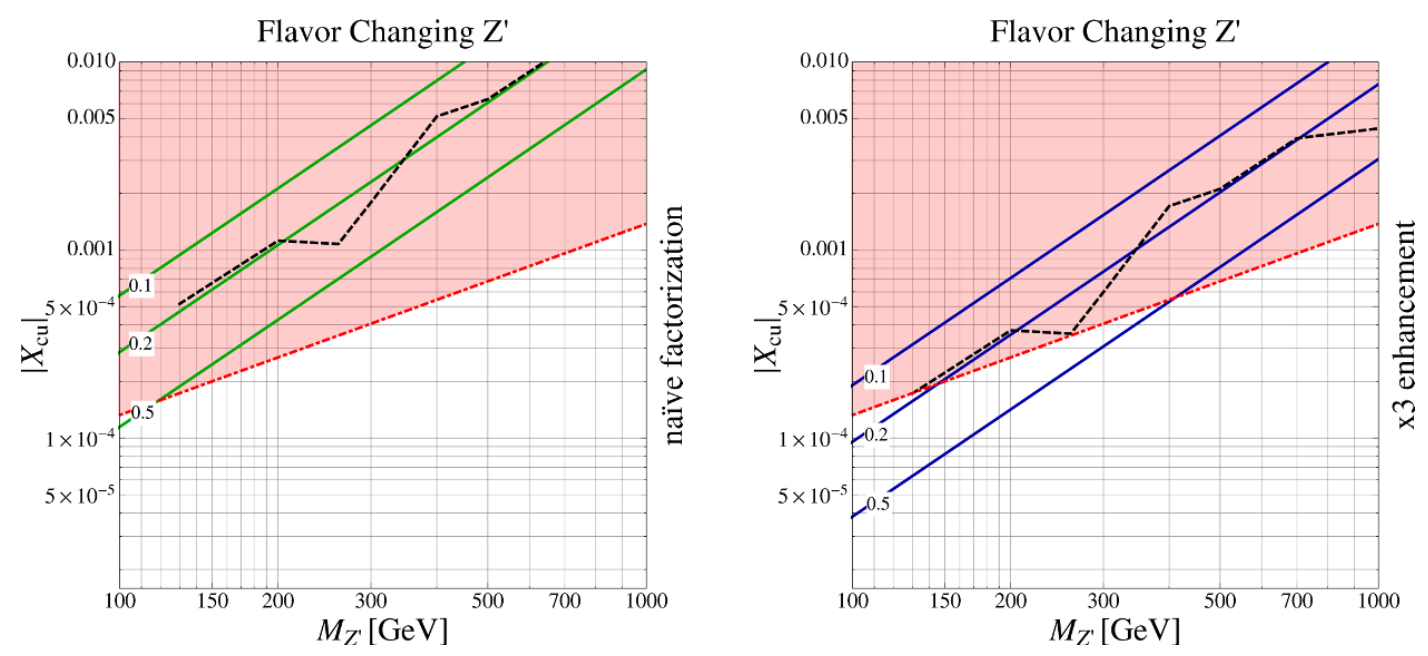

Figure 3. The $M_{Z^{\prime}}-\left|X_{c u}\right|$ plane, setting $\operatorname{Arg}\left(X_{c u}\right)=\pi / 2$. In the left plot, $\Delta A_{\mathrm{CP}}$ is evaluated in naïve factorization and in the right plot, we allow for an enhancement by a factor of 3 . Along the solid green and blue lines the NP contributions to $\Delta A_{\mathrm{CP}}$ match the world average. The different green and blue lines correspond to different common choices of the flavor conserving couplings $g_{u}=g_{d}=g_{L}$ as indicated. The black dashed line shows the constraint on the flavor conserving coupling from dijet searches. The red (dash-dotted) region is excluded by $D^{0}-\bar{D}^{0}$ mixing constraints.

Depending on whether the flavor conserving couplings of the $Z^{\prime}$ are to left-handed or right-handed quarks, the flavor changing $\bar{c} u Z^{\prime}$ coupling can induce tree level contributions to the Wilson coefficients $\tilde{C}_{5}^{(1)}, \tilde{C}_{3}^{(1)}$, and $\tilde{C}_{9}^{(1)}$

$$
\begin{aligned}
& \tilde{C}_{3}^{(1)}=\frac{\left(g_{u}+2 g_{d}\right)}{3} \frac{X_{c u}^{*}}{4 M_{Z^{\prime}}^{2}}, \\
& \tilde{C}_{9}^{(1)}=\frac{2\left(g_{u}-g_{d}\right)}{3} \frac{X_{c u}^{*}}{4 M_{Z^{\prime}}^{2}}, \\
& \tilde{C}_{5}^{(1)}=\frac{g_{L} X_{c u}^{*}}{4 M_{Z^{\prime}}^{2}} .
\end{aligned}
$$

If we assume $g_{u}=g_{d}$, then only contributions to $\tilde{C}_{3}^{(1)}$ and $\tilde{C}_{5}^{(1)}$ are generated. The contributions from $\tilde{C}_{3,5,9}^{(1)}$ to the $D \rightarrow K^{+} K^{-}$and $D \rightarrow \pi^{+} \pi^{-}$decay amplitudes are color suppressed. The contribution from $\tilde{C}_{5}^{(1)}$ is helicity enhanced.

The flavor changing $\bar{c} u Z^{\prime}$ coupling also induces tree level contributions to $D^{0}-\bar{D}^{0}$ mixing

$$
\tilde{C}_{1}^{(2) D}=\frac{\left(X_{c u}^{*}\right)^{2}}{2 M_{Z^{\prime}}^{2}} .
$$

We highlight the following point: as both the NP contributions to the $\Delta F=2$ mixing amplitude and the $\Delta F=1$ decay amplitudes are described by dimension 6 operators, they decouple with the NP mass squared. Yet while the $\Delta F=2$ amplitude is obviously proportional to the square of the flavor changing coupling, the $\Delta F=1$ amplitude is linearly proportional in this coupling. Correspondingly, the constraint from $D^{0}-\bar{D}^{0}$ mixing 
becomes more effective with heavier NP mass. Analogous arguments hold in all the other NP scenarios discussed in this work.

If the $Z^{\prime}$ boson has flavor changing couplings to left-handed quarks, tree level contributions to $K-\bar{K}$ mixing would be unavoidably generated since the $\bar{c}_{L} u_{L} Z^{\prime}$ and $\bar{s}_{L} d_{L} Z^{\prime}$ couplings are related by the CKM matrix due to $\mathrm{SU}(2)_{L}$ invariance. Since constraints coming from $K-\bar{K}$ mixing are considerably stronger than those coming from $D^{0}-\bar{D}^{0}$ mixing, we restrict ourselves to the $\bar{c}_{R} u_{R} Z$ coupling. As a result, 1-loop contributions to $\epsilon^{\prime} / \epsilon$ that can lead to constraints are also absent.

The dijet searches at hadron colliders set additional constraints on the model. In this paper, we consider the searches at UA2 [43], CDF [44] and CMS [45]. The UA2 collaboration probed the light dijet mass region, from $130 \mathrm{GeV}$ to $300 \mathrm{GeV}$, while the CDF search covers a dijet mass range from $260 \mathrm{GeV}$ to $1.4 \mathrm{TeV}$. A higher mass range, $1.0-4.1 \mathrm{TeV}$, is probed by the CMS experiment. There are other dijet searches from the $\mathrm{D} \varnothing$ [46] and ATLAS experiments [47]. The DØ collaboration analyzed $109 \mathrm{pb}^{-1}$ of data, however, while CDF analyzed $1.13 \mathrm{fb}^{-1}$, indicating the $\mathrm{D} \varnothing$ bound is less competitive than the one from CDF. The ATLAS bound is expected to be comparable with the CMS bound since both of the experiments analyzed $1 \mathrm{fb}^{-1}$ of data.

We simulate the $Z^{\prime}$ production using MadGraph 5 [48], and the width of the $Z^{\prime}$ is calculated with CompHEP [49], varying $g_{u}=g_{d}=g_{L}$. We compare the simulated cross section with the limit on dijet production from UA2 (figure 2 of [43]), CDF (table I of [44]) and CMS (table 1 of [45]). In calculating the bound, we ignore $X_{c u}$ since it is at least one order of magnitude smaller than $g_{u}, g_{d}$ and $g_{L}$.

The plots in figure 3 show the $M_{Z^{\prime}}-\left|X_{c u}\right|$ plane, setting $\operatorname{Arg}\left(X_{c u}\right)=\pi / 2$. In the left plot, $\Delta A_{\mathrm{CP}}$ is evaluated in the naïve factorization approach, while in the right plot we allow for an enhancement of the hadronic matrix elements by a factor of 3 . Along the green (solid, left plot) and blue (solid, right plot) lines, the NP contributions to $\Delta A_{\mathrm{CP}}$ match the world average. The different green or blue lines correspond to different choices of the flavor conserving couplings $g_{u}=g_{d}=g_{L}=0.1,0.2$, or 0.5 . The region below the black (dashed) line requires a flavor conserving coupling that is excluded by dijet searches. The red (dash-dotted) region is excluded by the constraints from $D^{0}-\bar{D}^{0}$ mixing. The choice $\operatorname{Arg}\left(X_{c u}\right)=\pi / 2$ corresponds to a maximal phase for the NP contributions to the $D \rightarrow K^{+} K^{-}$and $D \rightarrow \pi^{+} \pi^{-}$decays while simultaneously minimizing the constraint from $D^{0}-\bar{D}^{0}$ mixing. Choosing $\operatorname{Arg}\left(X_{c u}\right)=\pi / 3$ would lead to $\mathcal{O}(1)$ phases both in the decays and in $D^{0}-\bar{D}^{0}$ mixing, and the corresponding constraint would be more stringent by a factor of $\sim 2$. As expected, the $D^{0}-\bar{D}^{0}$ constraint becomes more effective with larger $Z^{\prime}$ mass.

We observe that even allowing for an enhancement in $\Delta A_{\mathrm{CP}}$ by a factor of three, the $D^{0}-\bar{D}^{0}$ mixing constraint in combination with dijet searches rules out a $Z^{\prime}$ as a tree level $\mathrm{NP}$ explanation for the measured $\Delta A_{\mathrm{CP}}$. We do not consider $Z^{\prime}$ masses below $100 \mathrm{GeV}$, which would be constrained from $Z-Z^{\prime}$ mixing [50], but the exact constraints would be model dependent and are beyond the scope of this work. 

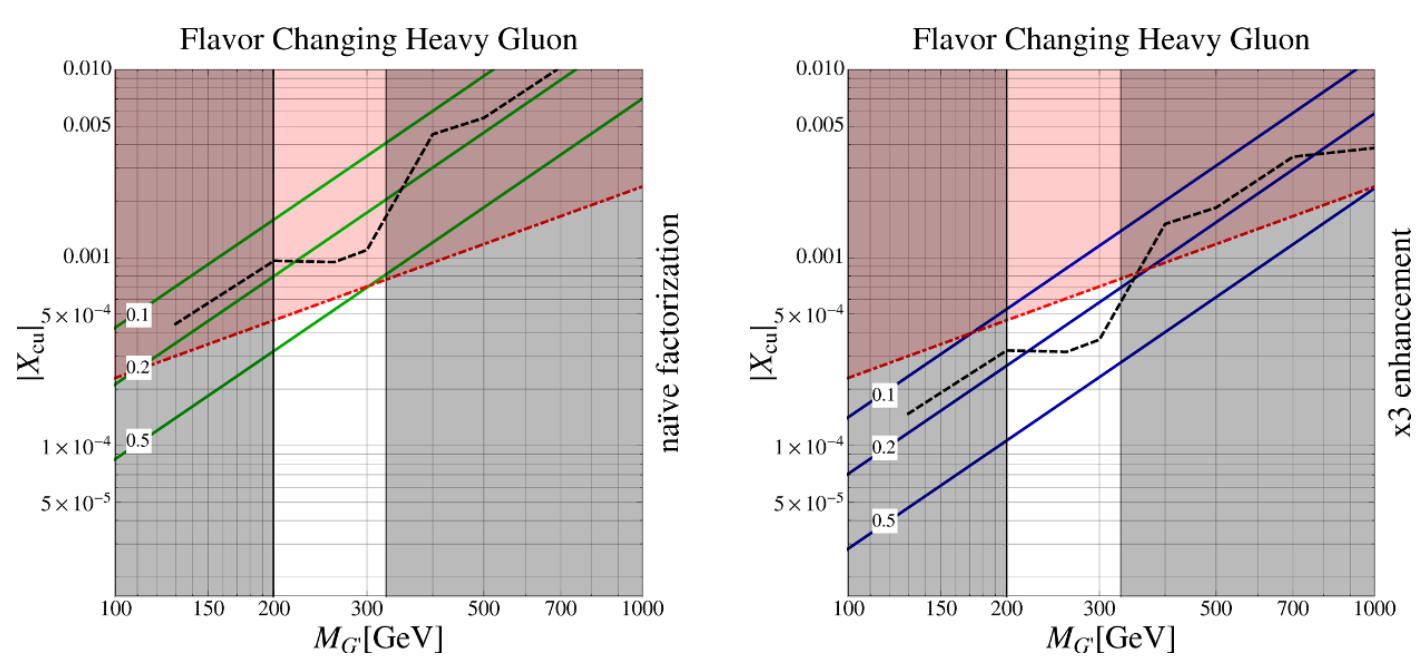

Figure 4. As figure 3 but in the model with flavor changing heavy gluon. The shaded vertical bands are excluded by dijet pair searches at ATLAS (left band) and CMS (right band).

\subsection{Flavor changing heavy gluon}

Heavy color octet vector bosons that have flavor changing couplings to SM quarks can arise, for example, in models with warped extra dimensions [51] and also in models of axigluons with family non-universal couplings [52]. We concentrate again on a direct tree level coupling between right-handed up and charm quarks

$$
\begin{aligned}
\mathcal{L}_{\text {int }}= & g_{L} \bar{u}_{L}^{i} \gamma^{\mu} T^{a} u_{L}^{i}\left(G^{\prime}\right)_{\mu}^{a}+g_{u} \bar{u}_{R}^{i} \gamma^{\mu} T^{a} u_{R}^{i}\left(G^{\prime}\right)_{\mu}^{a} \\
& +g_{L} \bar{d}_{L}^{i} \gamma^{\mu} T^{a} d_{L}^{i}\left(G^{\prime}\right)_{\mu}^{a}+g_{d} \bar{d}_{R}^{i} \gamma^{\mu} T^{a} d_{R}^{i}\left(G^{\prime}\right)_{\mu}^{a} \\
& +X_{c u} \bar{c}_{R} \gamma^{\mu} T^{a} u_{R}\left(G^{\prime}\right)_{\mu}^{a}+\text { h.c. }
\end{aligned}
$$

The flavor universal couplings $g_{u}, g_{d}$ and $g_{L}$ are free real parameters: the (small) flavor violating coupling $X_{c u}$ is a free, complex parameter.

The heavy gluon can generate tree level contributions to $\tilde{C}_{3,4,5,6}^{(1)}$ and $\tilde{C}_{9,10}^{(1)}$

$$
\begin{aligned}
\tilde{C}_{4}^{(1)} & =\frac{\left(g_{u}+2 g_{d}\right)}{3} \frac{X_{c u}^{*}}{8 M_{G^{\prime}}^{2}}, & \tilde{C}_{3}^{(1)} & =\frac{-1}{N_{c}} \tilde{C}_{4}^{(1)}, \\
\tilde{C}_{10}^{(1)} & =\frac{2\left(g_{u}-g_{d}\right)}{g_{u}+2 g_{d}} \tilde{C}_{4}^{(1)}, & \tilde{C}_{9}^{(1)} & =\frac{-1}{N_{c}} \tilde{C}_{10}^{(1)}, \\
\tilde{C}_{6}^{(1)} & =\frac{g_{L} X_{c u}^{*}}{8 M_{G^{\prime}}^{2}}, & \tilde{C}_{5}^{(1)} & =\frac{-1}{N_{c}} \tilde{C}_{6}^{(1)} .
\end{aligned}
$$

To generate $\tilde{C}_{3,4}^{(1)}$ and $\tilde{C}_{9,10}^{(1)}$, the $\mathrm{RH}$ flavor conserving coupling is required, while for $\tilde{C}_{5,6}^{(1)}$ the LH flavor conserving coupling is required. If we assume $g_{u}=g_{d}, \tilde{C}_{9,10}^{(1)}$ are absent. The Wilson coefficients $\tilde{C}_{3,5,9}^{(1)}$ are color suppressed, and furthermore, their contributions to the decay amplitudes are color suppressed. The contributions to the decay amplitudes from $\tilde{C}_{5}^{(1)}$ and $\tilde{C}_{6}^{(1)}$ are helicity enhanced. Due to the presence of $\tilde{C}_{6}^{(1)}$, we expect slightly larger $\mathrm{NP}$ contributions to $\Delta A_{\mathrm{CP}}$ than the $Z^{\prime}$ scenario. 
Similar to the models discussed before, the flavor changing $\bar{c} u G^{\prime}$ coupling also leads to tree level contributions to $D^{0}-\bar{D}^{0}$ mixing

$$
\tilde{C}_{1}^{(2) D}=\frac{1-N_{c}}{2 N_{c}} \frac{\left(X_{c u}^{*}\right)^{2}}{2 M_{G^{\prime}}^{2}}
$$

Compared to the $Z^{\prime}$ case, the contribution to $D^{0}-\bar{D}^{0}$ mixing is suppressed by a factor $\left|\left(1-N_{c}\right) /\left(2 N_{c}\right)\right|=1 / 3$. As discussed for the $Z^{\prime}$ case, we do not consider flavor changing couplings to left-handed quarks to avoid the stringent constraints from $K-\bar{K}$ mixing and $\epsilon^{\prime} / \epsilon$.

Collider constraints come again from dijet searches at the hadron colliders, and we evaluate them in a similar fashion to the $Z^{\prime}$ case. Additional constraints arise from recent results on four jet searches at the LHC, searching for pair production of dijet resonances. Pair production of the heavy gluon is fixed by QCD to good approximation and the production cross section depends only on the $G^{\prime}$ mass [53]. The mass range from $100 \mathrm{GeV}$ to $200 \mathrm{GeV}$ is covered by an ATLAS search using $34 \mathrm{pb}^{-1}$ [54] while a CMS search using $2.2 \mathrm{fb}^{-1}$ [55] starts at $320 \mathrm{GeV}$. The intermediate region from $200 \mathrm{GeV}$ to $320 \mathrm{GeV}$ is not considered in the CMS search because of the multijet trigger turn-on effects on the QCD background fit curve, which well models the QCD multijet background above $320 \mathrm{GeV}$. We simulate pair production of the $G^{\prime}$ resonance using Madgraph 5. We find that a $G^{\prime}$ mass in the ranges $100 \mathrm{GeV}<M_{G^{\prime}}<200 \mathrm{GeV}$ is excluded. Assuming the signal acceptance to be $3 \%$ (13\%), which corresponds to the lowest (highest) signal efficiency found in [55], we find that a $G^{\prime}$ mass from $320 \mathrm{GeV}$ up to $720 \mathrm{GeV}(1000 \mathrm{GeV})$ is excluded. We conservatively use the stronger bound derived from an acceptance of $13 \%$ in the following discussion.

The plots in figure 4 show the $M_{G^{\prime}}-\left|X_{c u}\right|$ plane with $\operatorname{Arg}\left(X_{c u}\right)=\pi / 2$ in order to minimize the constraint from $D^{0}-\bar{D}^{0}$ mixing. In the left plot, $\Delta A_{\mathrm{CP}}$ is evaluated in the naïve factorization approach, while in the right plot we allow for an enhancement by a factor of 3. The NP contributions to $\Delta A_{\mathrm{CP}}$ match the world average along the green (solid, left plot) and blue (solid, right plot) lines. The different green or blue lines correspond to different choices of the flavor conserving couplings $g_{u}=g_{d}=g_{L}=0.1,0.2$, or 0.5 . The region below the black (dashed) line requires a flavor conserving coupling that is excluded by dijet resonance searches. The red (dash-dotted) region is excluded by the constraints from $D^{0}-\bar{D}^{0}$ mixing. The vertical bands are excluded by the dijet pair searches at ATLAS (left band) and CMS (right band).

Because of different $\mathcal{O}(1)$ factors in the $D \rightarrow K^{+} K^{-}$and $D \rightarrow \pi^{+} \pi^{-}$decay amplitudes and $D^{0}-\bar{D}^{0}$ mixing compared to the $Z^{\prime}$, a heavy gluon appears slightly better suited to generate nonstandard effects in $\Delta A_{\mathrm{CP}}$. Yet only after allowing for an enhancement in $\Delta A_{\mathrm{CP}}$ by a factor of 3 can the combined constraints from $D^{0}-\bar{D}^{0}$ mixing and dijet searches be made compatible with the measured $\Delta A_{\mathrm{CP}}$. The corresponding corner of parameter space is characterized by light $G^{\prime}$ masses $M_{G^{\prime}} \lesssim 300 \mathrm{GeV}$. As the currently available results for dijet pair searches do not exclude the range between $200 \mathrm{GeV}$ and $320 \mathrm{GeV}$, a heavy gluon cannot be ruled out as a possible NP explanation of the observed $\Delta A_{\mathrm{CP}}$. If a heavy gluon is indeed responsible for the large value of $\Delta A_{\mathrm{CP}}$, indirect $\mathrm{CPV}$ in $D^{0}-\bar{D}^{0}$ mixing is also expected to be close to the current experimental bounds. 


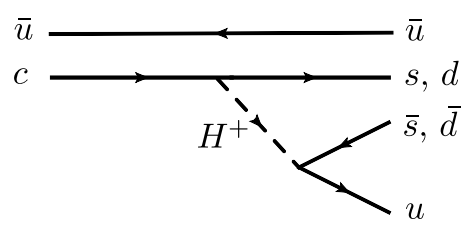

(a)

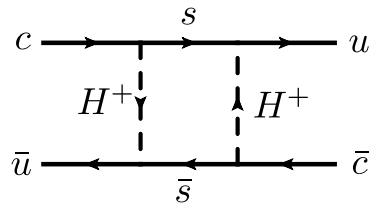

(b)

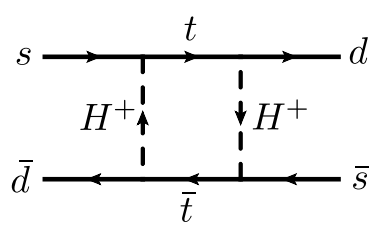

(c)

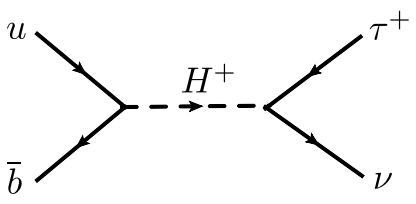

(d)

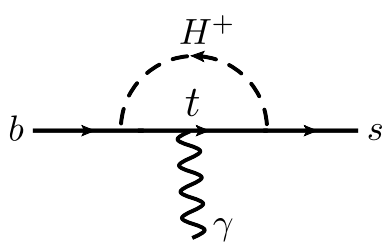

(e)

Figure 5. Example Feynman diagrams that contribute to (a) the $D \rightarrow K^{+} K^{-}$and $D \rightarrow \pi^{+} \pi^{-}$ decay amplitudes, (b) $D^{0}-\bar{D}^{0}$ mixing, (c) $K-\bar{K}$ mixing, (d) the $B^{+} \rightarrow \tau \nu$ decay and (e) the $B_{d} \rightarrow X_{s} \gamma$ decay in the discussed $2 \mathrm{HDM}$ with MFV.

\subsection{Charged vector boson}

We consider a new vector boson with charge \pm 1 that couples to right-handed up and down type quarks

$$
\mathcal{L}_{\text {int }}=g_{R} V_{i j}^{R} \bar{d}_{R}^{i} u_{R}^{j} W^{\prime-}+\text { h.c. },
$$

where $V_{i j}^{R}$ is a unitary mixing matrix, the analog of the CKM matrix in the right-handed sector. One possibility to introduce such a $W^{\prime}$ gauge boson is through an additional $\mathrm{SU}(2)_{R}$ gauge group [56]. Yet as long as the coupling structure in eq. (4.10) is realized, the exact implementation of the $W^{\prime}$ is of no relevance for the following discussion.

Tree level exchange of the $W^{\prime}$ gives contributions to the current-current Wilson coefficient $\tilde{C}_{1}^{(1) p}$. The corresponding operator $\tilde{O}_{1}^{(1) p}$ has the same hadronic matrix element as the SM operator $O_{1}^{(1) p}$. Therefore, tree level exchange of the $W^{\prime}$ cannot generate a direct $\mathrm{CP}$ asymmetry because there is no strong phase difference with respect to the LO SM contribution.

Loop level contributions, i.e. gluon penguins with $W^{\prime}$ loops, have a structure that is analogous to the SM penguin contribution. If we assume that the mass of the $W^{\prime}$ is larger than the mass of the SM $W$ boson, then the couplings $g_{R} V_{c b}^{R} V_{u b}^{R *}$ have to be considerably larger than the SM couplings $g V_{c b} V_{u b}^{*}$ in order to generate a sizable $\Delta A_{\mathrm{CP}}$. Such a $W^{\prime}$ would then lead to unacceptably large NP contributions to $B \rightarrow D$ and $B \rightarrow \pi$ transitions, and thus we will not consider this scenario any further.

\subsection{Two Higgs doublet model}

One of the simplest extensions of the SM scalar sector is the 2 Higgs doublet model (2HDM) (see [57] for a recent review). The most general couplings of the 2 Higgs bosons to SM fermions read

$$
\begin{aligned}
\mathcal{L}_{\mathrm{int}}= & Y_{u} \bar{Q} U H_{u}+Y_{d} \bar{Q} D H_{d}+Y_{\ell} \bar{L} E H_{d} \\
& +X_{u} \bar{Q} U H_{d}^{\dagger}+X_{d} \bar{Q} D H_{u}^{\dagger}+X_{\ell} \bar{L} E H_{u}^{\dagger}+\text { h.c. },
\end{aligned}
$$


where we suppress the flavor indices on the quark fields and the coupling matrices $X$ and $Y$. Generically, the neutral components of both Higgs bosons acquire a vev $\left\langle H_{u, d}^{0}\right\rangle=v_{u, d}$ and the fermion mass matrices receive contributions from both $Y$ and $X$. As $Y$ and $X$ are independent matrices in flavor space, the couplings in eq. (4.11) lead to flavor changing neutral Higgs vertices at tree level and are strongly constrained from meson mixing observables.

The most effective way to control flavor changing effects in the $2 \mathrm{HDM}$ is the Minimal Flavor Violation (MFV) ansatz [58, 59]. Applied to the 2HDM, the MFV assumption states that the "wrong" Higgs couplings $X$ can be expanded in powers of the Yukawa couplings $Y$

$$
\begin{aligned}
X_{u} & =\epsilon_{u} Y_{u}+\epsilon_{u}^{\prime} Y_{u} Y_{u}^{\dagger} Y_{u}+\epsilon_{u}^{\prime \prime} Y_{d} Y_{d}^{\dagger} Y_{u}+\ldots, \\
X_{d} & =\epsilon_{d} Y_{d}+\epsilon_{d}^{\prime} Y_{u} Y_{u}^{\dagger} Y_{d}+\epsilon_{d}^{\prime \prime} Y_{d} Y_{d}^{\dagger} Y_{d}+\ldots
\end{aligned}
$$

where the $\epsilon_{i}$ are free complex parameters. For simplicity, we will also assume $X_{\ell}=\epsilon_{\ell} Y_{\ell}$.

We stress that in this particular $2 \mathrm{HDM}$, there exists no preferred basis for the 2 Higgs doublets. The $\epsilon_{i}$ parameters as well as $\tan \beta=v_{u} / v_{d}$ are basis dependent and none of them separately are actually physical parameters (see [60] for a detailed discussion). In the following analysis, we fix a basis by setting $\epsilon_{d}=0$. In this basis, a large $\tan \beta$ can be approximately identified with the basis invariant enhancement of the coupling of the right handed strange quark to the charged Higgs with respect to its SM Yukawa coupling.

We now investigate the parameter space of this $2 \mathrm{HDM}$ with MFV in a basis with $\epsilon_{d}=0$. We work in the regime of large $\tan \beta$ and assume $\epsilon_{u}^{\prime \prime}, \epsilon_{d}^{\prime} \lll 1$ in order to ensure that tree level FCNCs are under control. Furthermore, we allow the parameters $\epsilon_{u}, \epsilon_{u}^{\prime}$ and $\epsilon_{d}^{\prime \prime}$ as well as $\epsilon_{\ell}$ to be $\mathcal{O}(1)$. As we will see below, in this region of parameter space, sizable nonstandard contributions to the $D \rightarrow K^{+} K^{-}$amplitude can arise, while the most important constraints can be kept under control.

In this scenario, tree level charged Higgs exchange, as shown in diagram (a) of figure 5, gives the dominant NP contribution to the $D \rightarrow K^{+} K^{-}$decay. For large values of $\tan \beta$ and assuming $\epsilon_{u} \sim \mathcal{O}(1)$ we find $^{2}$

$$
\tilde{C}_{S 1}^{(1)}=\frac{m_{c} m_{s}}{v^{2}} \epsilon_{u} \frac{\tan \beta}{1+\tilde{\epsilon}_{s} \tan \beta} \frac{V_{u s} V_{c s}^{*}}{M_{H^{ \pm}}^{2}},
$$

with $v^{2}=v_{u}^{2}+v_{d}^{2}=174^{2} \mathrm{GeV}^{2}$, and $\tilde{\epsilon}_{s} \equiv \epsilon_{d}^{\prime \prime} y_{s}^{2}$. For large $\tan \beta$ and $\epsilon_{d}^{\prime \prime} \sim \mathcal{O}(1)$, we find $\tilde{\epsilon}_{s} \sim 10^{-3}$, such that $\tilde{\epsilon}_{s}$ only becomes relevant for extremely large $\tan \beta$. The parameter $\epsilon_{u}$ simultaneously lifts the $1 / \tan \beta$ suppression of the $\bar{c}_{R} s_{L} H^{+}$vertex and provides a source of CPV.

The 2HDM with MFV have been thoroughly studied in the literature (see [59, 61-64]) and various constraints have been identified. In the following, we discuss the most important constraints:

(i) Direct searches at LEP for a charged Higgs give the bound $M_{H^{ \pm}} \gtrsim 80 \mathrm{GeV}$ [65].

\footnotetext{
${ }^{2}$ Note that this expression as well as the ones given below are not basis invariant. They only hold in bases where the same conditions on the $\epsilon_{i}$ and $\tan \beta$ hold, under which they were derived.
} 
(ii) The $B^{+} \rightarrow \tau^{+} \nu$ and $K^{+} \rightarrow \mu^{+} \nu$ decays are known to be important low energy probes of extended Higgs sectors. Combining the experimental results from Belle [66, 67] and $\mathrm{BaBar}[68,69]$ on $\operatorname{Br}(B \rightarrow \tau \nu)$ with a conservative SM prediction based on $\left|V_{u b}\right|=(3.89 \pm 0.44) \times 10^{-3}[10]$ and $f_{B^{+}}=196.9 \pm 8.9 \mathrm{MeV}[70]$

$$
\operatorname{Br}(B \rightarrow \tau \nu)_{\mathrm{SM}}=(1.04 \pm 0.25) \times 10^{-4},
$$

we find

$$
R_{B \tau \nu}=\frac{\operatorname{Br}(B \rightarrow \tau \nu)_{\exp }}{\operatorname{Br}(B \rightarrow \tau \nu)_{\mathrm{SM}}}=1.58 \pm 0.32 .
$$

In our 2HDM, tree level exchange of a charged Higgs (see diagram (d) in figure 5) results in

$$
R_{B \tau \nu}=\left|1-\frac{m_{B}^{2}}{M_{H^{ \pm}}^{2}} \frac{\tan \beta}{1+\tilde{\epsilon}_{b} \tan \beta} \frac{1}{\epsilon_{\ell}}\right|^{2},
$$

where we defined $\tilde{\epsilon}_{b} \equiv \epsilon_{d}^{\prime \prime} y_{b}^{2}$. For large $\tan \beta$ and $\epsilon_{d}^{\prime \prime} \sim \mathcal{O}(1)$, one typically has $\tilde{\epsilon}_{b} \sim 10^{-2}-10^{-1}$. The factors $\tilde{\epsilon}_{b}$ and $\epsilon_{\ell}$ keep the $\bar{b}_{R} u_{L} H^{ \pm}$and $\bar{\tau}_{R} \nu_{L} H^{ \pm}$couplings small for large $\tan \beta$ and the experimental constraint from $B^{+} \rightarrow \tau^{+} \nu$ is easily avoided, provided $\operatorname{sign}\left(\epsilon_{\ell}\right)=-1$. In fact, for such choices of parameters, the $\sim 2 \sigma$ discrepancy between the SM prediction and the experimental result for $\operatorname{Br}(B \rightarrow \tau \nu)$ is reduced.

For the observable $R_{\ell 23}$ [71], which is sensitive to charged Higgs contributions to the $K \rightarrow \mu \nu$ decay, we find

$$
R_{\ell 23}=\left|1-\frac{m_{K}^{2}}{M_{H^{ \pm}}^{2}} \frac{\tan \beta}{1+\tilde{\epsilon}_{s} \tan \beta} \frac{1}{\epsilon_{\ell}}\right| .
$$

From the experimental side, one has [71]

$$
R_{\ell 23}=0.999 \pm 0.007,
$$

which, in our framework, leads only to constraints for extremely large $\tan \beta$.

(iii) Charged Higgs loops lead to contributions to $D^{0}-\bar{D}^{0}$ and $K-\bar{K}$ mixing (see diagrams (b) and (c) of figure 5). In the considered scenario, we find the following dominant NP contributions

$$
\begin{aligned}
C_{1}^{(2) D} \simeq & \frac{-1}{16 \pi^{2}} \frac{m_{s}^{4}}{v^{4}} \frac{\left(V_{c s} V_{u s}^{*}\right)^{2}}{8 M_{H^{ \pm}}^{2}} \frac{\tan ^{4} \beta}{\left|1+\tilde{\epsilon}_{s} \tan \beta\right|^{4}}, \\
C_{1}^{(2) K} \simeq & \frac{-1}{16 \pi^{2}} \frac{m_{t}^{4}}{v^{4}} \frac{\left(V_{t s} V_{t d}^{*}\right)^{2}}{4 M_{H^{ \pm}}^{2}} \\
& \left(\left|\tilde{\epsilon}_{t}\right|^{4} h_{1}\left(x_{t}\right)+\left|\tilde{\epsilon}_{t}\right|^{2} h_{2}\left(x_{t}, x_{W}\right)\right),
\end{aligned}
$$

with $x_{t}=m_{t}^{2} / M_{H^{ \pm}}^{2}, x_{W}=M_{W}^{2} / M_{H^{ \pm}}^{2}, \tilde{\epsilon}_{t} \equiv \epsilon_{u}+\epsilon_{u}^{\prime} y_{t}^{2}$, and the analytical expressions for the loop functions $h_{1}$ and $h_{2}$ are given in appendix C. The expression for $C_{1}^{(2) D}$ is suppressed by four powers of the strange quark mass and is only relevant for very large $\tan \beta .^{3}$ The Wilson coefficient $C_{1}^{(2) K}$ arises from box diagrams including both

\footnotetext{
${ }^{3}$ The corresponding contribution from a bottom quark loop is strongly Cabibbo suppressed and turns out to be much smaller.
} 
one $W^{ \pm}$and one $H^{ \pm}$insertion as well as diagrams with two $H^{ \pm}$insertions. Its contribution to kaon mixing can be relevant for $\tilde{\epsilon}_{t} \simeq \mathcal{O}(1)$. Yet even for $\left|\tilde{\epsilon}_{t}\right| \simeq 0.5$, the constraint on the charged Higgs mass is as low as the bound from direct searches $M_{H^{ \pm}} \gtrsim 80 \mathrm{GeV}$.

(iv) As shown in diagram (e) of figure 5, the $B_{d} \rightarrow X_{s} \gamma$ decay also receives 1-loop charged Higgs contributions. As is well known, the good agreement of the experimental data and the SM prediction of its branching ratio leads to the constraint $M_{H^{ \pm}} \gtrsim 300 \mathrm{GeV}$ in a 2HDM of type II [72]. This bound, however, does not apply in the model considered here. For the ratio of the $b \rightarrow s \gamma$ amplitudes in our 2HDM with MFV model to the $2 \mathrm{HDM}$ of type II, we find, to a good approximation,

$$
\frac{A(b \rightarrow s \gamma)_{\mathrm{MFV}}}{A(b \rightarrow s \gamma)_{\mathrm{II}}}=\frac{\tilde{\epsilon}_{t} \tan \beta}{1+\tilde{\epsilon}_{b} \tan \beta}+\left|\tilde{\epsilon}_{t}\right|^{2} y\left(x_{t}\right)
$$

where $y\left(x_{t}\right)$ is a function of $x_{t}=m_{t}^{2} / M_{H^{ \pm}}$and is $\mathcal{O}(1)$. We point out that because of the $\tilde{\epsilon}_{t}$ and $\tilde{\epsilon}_{b}$ factors, the $b \rightarrow s \gamma$ amplitude can be complex, which relaxes the $B_{d} \rightarrow$ $X_{s} \gamma$ constraint considerably [73]. Still, charged Higgs masses as low as the constraint from direct searches require $\tilde{\epsilon}_{t}=\epsilon_{u}+\epsilon_{u}^{\prime} y_{t}^{2} \ll 1$, which implies a considerable amount of fine tuning.

(v) Complex $\epsilon_{i}$ parameters also lead to contributions to electric dipole moments (EDMs) (see $[61,62]$ ). While a detailed study of EDMs is beyond the scope of this work, we mention that in the studied framework, the contributions to the EDMs depend on the parameters $\tilde{\epsilon}_{t}$ and $\tilde{\epsilon}_{b}$. Therefore, by allowing for a certain amount of fine tuning, EDM constraints do not exclude sizable CP violating effects in the $D \rightarrow K^{+} K^{-}$ decay that depend mainly on $\epsilon_{u}$.

(vi) Charged Higgses are also constrained by bounds on the branching ratio of the top quark decay $t \rightarrow H^{+} b$. ATLAS and CMS obtained bounds at the level of $\operatorname{Br}(t \rightarrow$ $\left.H^{+} b\right) \lesssim 5 \%$ under the assumption $\operatorname{Br}\left(H^{-} \rightarrow \tau \nu\right)=1$ [74]. CDF and DØ also give bounds considering the $H \rightarrow c s$ final state. These bounds are at the level of $\operatorname{Br}\left(t \rightarrow H^{+} b\right) \lesssim 10 \%-20 \%[75,76]$. In our setup, the ratio of the $t \rightarrow H^{+} b$ and $t \rightarrow W b$ branching ratios is given by

$$
\begin{aligned}
\frac{\Gamma_{t H b}}{\Gamma_{t W b}}= & \frac{1-\left(m_{H} / m_{t}\right)^{2}}{1-\left(m_{W} / m_{t}\right)^{2}} \frac{1}{m_{W}^{2} m_{t}^{2}+m_{t}^{4}-2 m_{W}^{4}} \\
\times & {\left[\left(m_{t}^{2}\left|\tilde{\epsilon}_{t}\right|^{2}+\frac{m_{b}^{2} \tan ^{2} \beta}{\left|1+\tilde{\epsilon}_{b} \tan \beta\right|^{2}}\right)\left(m_{t}^{2}-m_{H}^{2}\right)\right.} \\
& \left.\quad+4 \operatorname{Re}\left(\frac{\tilde{\epsilon}_{t} \tan \beta}{1+\tilde{\epsilon}_{b} \tan \beta}\right) m_{t}^{2} m_{b}^{2}\right]
\end{aligned}
$$

For the parameter choices detailed below, we find the charged Higgs branching ratio into $\tau \nu$ does not exceed $10 \%$ due to the strongly enhanced couplings to strange quarks. Also, since $\operatorname{Br}\left(t \rightarrow H^{ \pm} b\right) \lesssim 10 \%$, top decays do not lead to constraints for our choice of parameters. 


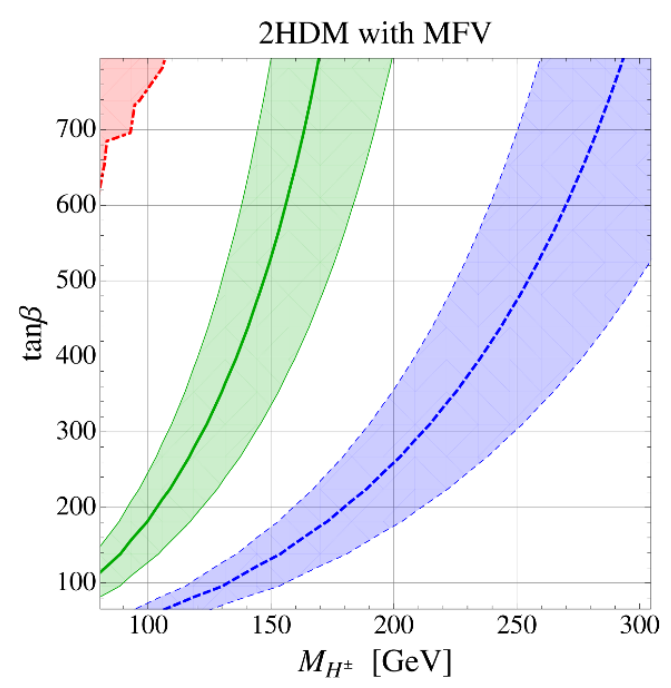

Figure 6. Regions in the $M_{H^{ \pm}}-\tan \beta$ plane compatible with the data on $\Delta A_{\mathrm{CP}}$, wA at the $1 \sigma$ level in the discussed 2HDM. In a basis with $\epsilon_{d}=0$, we set $\epsilon_{u}=i, \tilde{\epsilon}_{t}=0.05 i, \epsilon_{\ell}=-1$ and chose $\epsilon_{d}^{\prime \prime}$ such that $\tilde{\epsilon}_{s}=10^{-3}$. All other $\epsilon_{i}$ are set to zero. The green (solid) band corresponds to the expressions for the decay amplitude in naïve factorization, blue (dashed) band assumes an enhancement of the hadronic matrix elements by a factor of 3 . The red (dash-dotted) region is excluded by $D^{0}-\bar{D}^{0}$ mixing constraints.

(vii) Additional constraints on the $2 \mathrm{HDM}$ can come from direct searches for neutral Higgs bosons. The relation between the charged and the neutral Higgs bosons, however, depends on the details of the Higgs potential. For simplicity we assume a Higgs potential such that there is one neutral Higgs boson $h$ that has SM-like couplings to gauge bosons and fermions. Consequently, the other two bosons $H$ and $A$ do not couple to gauge bosons and have couplings to bottom quarks that are enhanced by $\tan \beta /\left(1+\tilde{\epsilon}_{b} \tan \beta\right)$. While the masses of the Higgs bosons are in principle free parameters, we will assume $M_{H} \simeq M_{A} \simeq M_{H^{ \pm}}$to avoid constraints from electroweak precision observables. Constraints can arise from neutral Higgs boson searches in the $H \rightarrow \tau \tau$ final state at ATLAS and CMS [77], as well as from searches at DØ and $\mathrm{CDF}$ in the $H \rightarrow b b$ final state $[78,79]$.

In the following, we discuss a benchmark scenario that avoids all considered constraints but allows for nonstandard values for $\Delta A_{\mathrm{CP}}$. We set $\epsilon_{u}=i, \tilde{\epsilon}_{t}=0.05 i, \epsilon_{\ell}=-1$, and chose $\epsilon_{d}^{\prime \prime}$ such that $\tilde{\epsilon}_{s}=10^{-3}$. All other $\epsilon_{i}$ are set to zero. Figure 6 shows the regions in the $M_{H^{ \pm}}-\tan \beta$ plane compatible with the data for $\Delta A_{\mathrm{CP}}$, wA at the $1 \sigma$ level. The green (solid) band is obtained using the expressions for the decay amplitude in naïve factorization. The blue (dashed) band assumes an enhancement of the hadronic matrix elements by a factor of 3. The red (dash-dotted) region in the upper left corner is excluded by $D^{0}-\bar{D}^{0}$ mixing constraints.

We observe that sizable NP contributions to the direct CP asymmetry are only possible for small charged Higgs masses and large values of $\tan \beta \sim \mathcal{O}(100)$ and larger. It is important to note that such large values for $\tan \beta$ are not in conflict with a requirement 


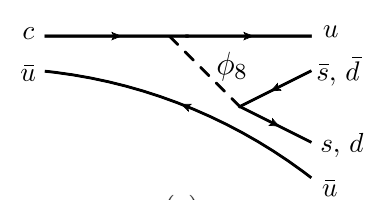

(a)

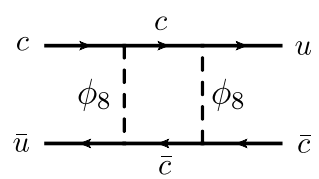

(b)

Figure 7. Example Feynman diagrams that contribute to (a) the $D \rightarrow K^{+} K^{-}$and $D \rightarrow \pi^{+} \pi^{-}$ decay amplitudes and (b) $D^{0}-\bar{D}^{0}$ mixing in the scalar octet model.

of perturbative Yukawa couplings. Indeed, due to the $\tilde{\epsilon}_{b}$ (we find $0.03<\tilde{\epsilon}_{b}<0.09$ in the considered region of $\tan \beta$ ) and $\epsilon_{\ell}$ factors, the bottom and tau Yukawa remain well below 1. ${ }^{4}$ Extremely large values of $\tan \beta$ are not stable under radiative corrections. Generically, 1-loop corrections to the Higgs potential lead to $1 / \tan \beta \sim 1 /(4 \pi)^{2}$. Values of $\tan \beta \simeq$ few $\times 100$, however, seem a reasonable possibility.

The shown region of parameter space is not significantly constrained by any of the considered bounds. In particular, the bound from $\operatorname{Br}\left(B_{d} \rightarrow X_{s} \gamma\right)$ is avoided because of the small $\tilde{\epsilon}_{t}$. As stated before, the branching ratio of the charged Higgs into $\tau \nu$ does not exceed $10 \%$ due to the strongly enhanced couplings to strange quarks, and in addition, constraints from top decays are satisfied since $\operatorname{Br}\left(t \rightarrow H^{ \pm} b\right) \lesssim 10 \%$. Similarly, we find that the branching ratios of the neutral Higgs bosons to $\tau \tau$ are tiny, $\mathcal{O}(0.1 \%)$, and the stringent bounds from ATLAS and CMS [77] are easily avoided. Finally, due to the $\tilde{\epsilon}_{b}$ factor that controls the size of the $\bar{b} b H$ and $\bar{b} b A$ couplings, the $H \rightarrow b b$ searches at Tevatron $[78,79]$ do not lead to constraints. We expect that the collider phenomenology of this large parameter space will focus on a combination of complementary probes, including tests for non-SM Yukawa couplings of the light Higgs boson and heavy flavor searches for pairs of dijet resonances. A complete phenomenological study of this $2 \mathrm{HDM}$ with MFV is left for future work.

We stress that the $2 \mathrm{HDM}$ with MFV can only significantly affect the direct CP asymmetry in $D \rightarrow K^{+} K^{-}$. New Physics effects in $A_{\mathrm{CP}}\left(\pi^{+} \pi^{-}\right)$are strongly suppressed by $m_{d} / m_{s}$ and negligibly small. The considered model also does not lead to large nonstandard effects in indirect CPV in $D^{0}-\bar{D}^{0}$ mixing. These statements are not necessarily true if the couplings of the Higgses radically departure from the MFV ansatz (see [24] for a related study). Even though such models will generically have flavor changing neutral Higgs interactions, they can in principle be made compatible with low energy flavor constraints. A detailed study of such setups, however, is beyond the scope of this work.

\subsection{Scalar octet}

Couplings analogous to the ones in eq. (4.11) are also possible if the second scalar $\mathrm{SU}(2)_{L}$ doublet is a color octet instead of a color singlet [82]. We write

$$
\begin{aligned}
\mathcal{L}_{\text {int }}= & Y_{u} \bar{Q} U H+Y_{d} \bar{Q} D H^{\dagger} \\
& +X_{u} \bar{Q} T^{a} U \phi_{8}^{a}+X_{d} \bar{Q} T^{a} D \phi_{8}^{a \dagger}+\text { h.c. }
\end{aligned}
$$

\footnotetext{
${ }^{4}$ The phenomenology of generating appropriate bottom and tau masses in the very large $\tan \beta$ regime in the context of supersymmetric models was studied in [80, 81]. We remark however that the considered scenario cannot be realized in the MSSM where the $\epsilon_{i}$ factors are only loop induced and not much larger than $10^{-2}$.
} 
where $H$ is the SM Higgs and $\phi_{8}^{a}, a=1, \ldots 8$, is the color octet. Since a color octet must not aquire a vev, the quark masses are entirely provided by the SM Higgs, and the Yukawa couplings $Y$ are given by their SM values. As in the case of the 2HDM, we will first assume that the couplings $X$ have the MFV structure

$$
\begin{aligned}
X_{u} & =\zeta_{u} Y_{u}+\zeta_{u}^{\prime} Y_{u} Y_{u}^{\dagger} Y_{u}, \\
X_{d} & =\zeta_{d} Y_{d}+\zeta_{d}^{\prime} Y_{d} Y_{u}^{\dagger} Y_{u} .
\end{aligned}
$$

We can neglect higher powers of the down-type Yukawa couplings in the expansion because in the considered scenario they are fixed to their small SM values. This automatically ensures that there are no tree level flavor changing interactions of the neutral component of the scalar octet with up quarks. Furthermore, the interactions of the charged component of the scalar octet cannot lead to tree level contributions to the $D \rightarrow K^{+} K^{-}$amplitude in naïve factorization, as the two quarks that couple to the color octet cannot hadronize into a kaon or pion, which are color singlets. Contributions to the $D \rightarrow K^{+} K^{-}$decay can, however, come from annihilation topologies involving the charged octet state. Even though such contributions are formally suppressed by $1 / m_{c}$, they can be sizable in $D$ meson decays. Nonetheless, we now consider departures from the MFV ansatz for the couplings and come back to the MFV framework at the end of the section. In particular, we consider the following (small) non-MFV entry in the $X_{u}$ coupling matrix in the basis where the quark Yukawa couplings are diagonal

$$
\left[X_{u}\right]_{12}=\zeta_{u} y_{c} X_{c u},
$$

with $X_{c u} \sim V_{c s} V_{u s}^{*} \sim \lambda \sim 0.2$. In contrast to the MFV interactions, the above term leads to a flavor changing neutral current $u_{L} c_{R} \phi_{8}^{0}$ coupling and, due to $\mathrm{SU}(2)_{L}$ invariance, to a $\mathcal{O}(1)$ correction of the $d_{L} c_{R} \phi_{8}^{ \pm}$coupling

$$
\begin{aligned}
\mathcal{L}_{\text {int }}= & \zeta_{u} y_{c} X_{c u} \bar{u}_{L} T^{a} c_{R} \phi_{8}^{0 a} \\
& +\zeta_{u} y_{c}\left(V_{c d}^{*}+X_{c u} V_{u d}^{*}\right) \bar{d}_{L} T^{a} c_{R} \phi_{8}^{-a}+\text { h.c. } .
\end{aligned}
$$

The tree level exchange of the neutral component of the scalar octet, as shown in diagram (a) of figure 7, then leads to the following contributions to the Wilson coefficients,

$$
\begin{aligned}
& \tilde{C}_{S 1}^{(1)}=\frac{m_{c} m_{s}}{v^{2}} \zeta_{u} \zeta_{d} \frac{X_{c u}}{4 M_{\phi_{8}}^{2}}, \\
& \tilde{C}_{S 2}^{(1)}=-\frac{4}{N_{c}} \tilde{C}_{T 1}^{(1)}=4 \tilde{C}_{T 2}^{(1)}=-\frac{1}{N_{c}} \tilde{C}_{S 1}^{(1)} .
\end{aligned}
$$

Even though the tensor operators do not contribute to the $D \rightarrow K^{+} K^{-}$decay, we include the corresponding Wilson coefficients here because they mix with the scalar operators under renormalization.

Tree level contributions to $D^{0}-\bar{D}^{0}$ mixing from the exchange of the neutral complex scalar $\phi_{8}^{0}$ can only arise if both the flavor changing couplings $\bar{u}_{L} c_{R} \phi_{8}^{0}$ and $\bar{c}_{L} u_{R} \phi_{8}^{0}$ are present simultaneously. Thus, in our setup, contributions to $D^{0}-\bar{D}^{0}$ mixing first arise at 
the loop level. The dominant contribution comes from a charm quark loop, as shown in diagram (b) of figure 7 ,

$$
C_{1}^{(2) D}=\frac{1}{16 \pi^{2}} \frac{N_{c}^{3}-2 N_{c}+1}{4 N_{c}^{2}} \frac{m_{c}^{4}}{v^{4}} \frac{X_{c u}^{2}}{8 M_{\phi_{8}}^{2}}\left|\zeta_{u}\right|^{4} .
$$

Similar to the situation discussed in the context of the 2HDM above, other flavor changing processes like kaon mixing or $B_{d} \rightarrow X_{s} \gamma$ do not directly constrain $\zeta_{u}$ or $\zeta_{d}$ as they involve couplings to right-handed top and bottom quarks that are proportional to $\zeta_{t}=\zeta_{u}+\zeta_{u}^{\prime} y_{t}^{2}$ and $\zeta_{b}=\zeta_{d}+\zeta_{d}^{\prime} y_{t}^{2}$, respectively. Keeping these couplings small to avoid constraints from perturbativity and, in particular from $B_{d} \rightarrow X_{s} \gamma$, requires a considerable amount of fine tuning. On the other hand, since color octets cannot couple to leptons, the constraints from $B \rightarrow \tau \nu$ and $K \rightarrow \mu \nu$ are automatically avoided.

Constraints from electroweak precision observables are under control, provided all components of the scalar octet have approximately the same mass and are heavier than $100 \mathrm{GeV}[83]$.

There has been extensive work done on collider constraints on scalar octets $[84,85]$. Because of the small couplings to light quarks in the considered framework, single production of scalar octets is strongly suppressed, and dijet resonance searches at the hadron colliders give no significant constraints in the considered region of parameter space. Instead, the main collider constraints on the scalar octet doublet come from the dijet pair searches at the LHC. We simulate the production of scalar octet pairs (including production of neutral pairs and charged pairs) using MadGraph 5 and compare the obtained cross sections with the bounds set by ATLAS [54] and CMS [55]. We find that the region from $100 \mathrm{GeV}$ to $200 \mathrm{GeV}$ that is covered by the ATLAS search is fully excluded. The CMS search excludes scalar octet masses from $320 \mathrm{GeV}$ until approximately $550 \mathrm{GeV}$ for $13 \%$ acceptance (see section 4.3 for comments regarding the $200-320 \mathrm{GeV}$ region). As our scalar octets decay predominantly to $b$ quarks, searches for final states with multiple $b$ jets at Tevatron can potentially lead to constraints. Using the CDF result [86], the authors of [87] find that scalar octet masses of $M_{\phi_{8}} \lesssim 200 \mathrm{GeV}$ are excluded. Updated results from CDF [79] and DØ [78] do not give significantly improved bounds on the corresponding cross sections.

In figure 8 , we show the regions in the $M_{\phi_{8}}-\zeta_{u}$ plane that are compatible with the data for $\Delta A_{\mathrm{CP}}$, wA at the $1 \sigma$ level, assuming $\zeta_{u}=\zeta_{d} / 2$ real and setting $X_{c u}=\left|V_{c s} V_{u s}^{*}\right| e^{i \pi / 2}$. The green (solid) band corresponds to the expressions for the decay amplitude obtained in naïve factorization, and the blue (dashed) band assumes an enhancement of the hadronic matrix elements by a factor of 3 . The red (dash-dotted) region is excluded by $D^{0}-\bar{D}^{0}$ mixing constraints and the vertical bands are excluded by the dijet pair searches at ATLAS (left band) and CMS (right band). As expected, constraints from $D^{0}-\bar{D}^{0}$ mixing allow for a sizable $\Delta A_{\mathrm{CP}}$ only for small scalar masses. Given the constraints from the dijet pair searches at LHC, only a small window with octet masses between $200 \mathrm{GeV}$ and $320 \mathrm{GeV}$ and large values for $\zeta_{u, d} \gtrsim 20$ remains where significant values for $\Delta A_{\mathrm{CP}}$ can be generated by the scalar octet with couplings close to the MFV ansatz without assuming any enhancement of the hadronic matrix elements. Scalar octets heavier than the CMS bound of $M_{\phi_{8}} \simeq$ 


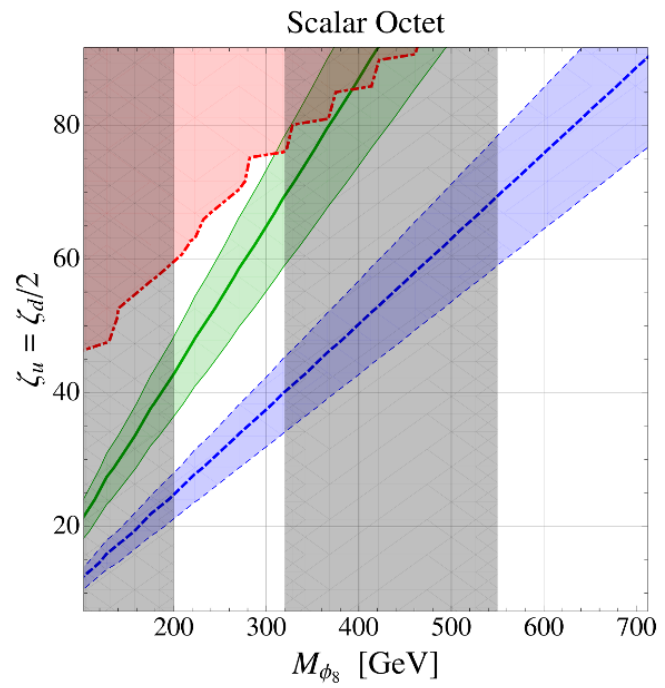

Figure 8. Regions in the $M_{\phi_{8}}-\zeta_{u}$ plane compatible with the data on $\Delta A_{\mathrm{CP}}$, wA at the $1 \sigma$ level in the scalar octet model, assuming $\zeta_{u}=\zeta_{d} / 2$ real and setting $X_{u c}=\left|V_{c s} V_{u s}^{*}\right| e^{i \pi / 2}$. The green (solid) band corresponds to the expressions for the decay amplitude obtained in naïve factorization, the blue (dashed) band assumes an enhancement of the hadronic matrix elements by a factor of 3 . The red (dash-dotted) region is excluded by $D^{0}-\bar{D}^{0}$ mixing constraints. The vertical shaded bands are excluded by dijet pair searches at LHC.

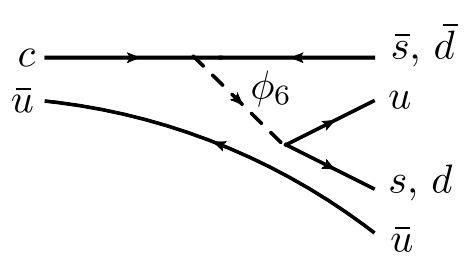

(a)

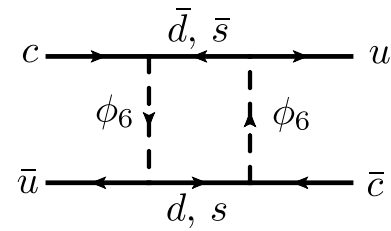

(b)

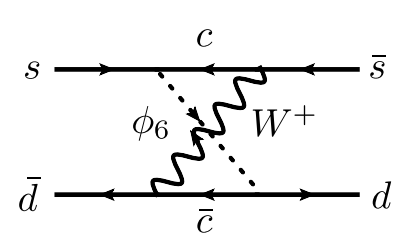

(c)

Figure 9. Example Feynman diagrams that contribute to (a) the $D \rightarrow K^{+} K^{-}$and $D \rightarrow \pi^{+} \pi^{-}$ decay amplitudes, (b) $D^{0}-\bar{D}^{0}$ mixing and (c) $K-\bar{K}$ mixing in the discussed diquark model.

$550 \mathrm{GeV}$ can lead to nonstandard values for $\Delta A_{\mathrm{CP}}$ only for extremely large $\zeta_{u, d} \gtrsim 60$ and assuming the hadronic matrix elements to be 3 times the naïve factorization estimate. The same conclusion holds for the pure MFV setup discussed at the beginning of the section, provided that the annihilation contribution with the charged scalar exchange is of similar size as the naïve factorization contribution from the flavor changing neutral scalar.

Both in the MFV and the quasi-MFV setup, the scalar octet can only significantly affect the direct $\mathrm{CP}$ asymmetry in $D \rightarrow K^{+} K^{-}$. New Physics effects in $A_{\mathrm{CP}}\left(\pi^{+} \pi^{-}\right)$are strongly suppressed by $m_{d} / m_{s}$ and negligibly small. As in the case of the $2 \mathrm{HDM}$, this is not necessarily the case if the octet couplings are allowed to deviate more radically from MFV. We expect collider searches in the multi- $b$ jet final state, as discussed in [84, 85], to provide the best sensitivity to this model. 


\subsection{Scalar diquarks}

Numerous variations of scalar diquarks can have renormalizable couplings to the SM quarks and lead to an interesting flavor phenomenology $[88,89]$. Here, we consider a diquark that has quantum numbers such that it leads to contributions to meson mixing only at the loop level, but contributes to the $D \rightarrow K^{+} K^{-}$and $D \rightarrow \pi^{+} \pi^{-}$decay amplitudes at tree level in naïve factorization. One such possibility is a scalar that transforms as $\overline{6}$ under SU(3), as a singlet under $\mathrm{SU}(2)_{L}$, and has hypercharge $-1 / 3 .{ }^{5}$ Such a diquark can couple to right-handed up and down type quarks,

$$
\mathcal{L}_{\text {int }}=X_{i j} \bar{U}_{i}^{c \alpha} D_{j}^{\beta} \phi_{6}^{\alpha \beta}+\text { h.c. },
$$

where $i, j$ are flavor indices and the diquark $\phi_{6}$ is symmetric in the color indices $\phi_{6}^{\alpha \beta}=\phi_{6}^{\beta \alpha}$. The couplings $X_{i j}$ are a source of flavor violation and in general free complex parameters. We remark that the considered diquark can also couple to left-handed quarks. Considering couplings to left- and right-handed quarks, however, simultaneously induces left-right mixing $\Delta F=2$ operators, resulting in very severe constraints from $D$ and $K$ meson mixing. Therefore, we concentrate on couplings to the right-handed quarks only.

The tree level contributions of the diquark to the $D \rightarrow K^{+} K^{-}$and $D \rightarrow \pi^{+} \pi^{-}$decay amplitudes are shown in diagram (a) of figure 9 and read

$$
\tilde{C}_{1}^{(1) p}=\tilde{C}_{2}^{(1) p}=\frac{X_{c p} X_{u p}^{*}}{16 M_{\phi_{6}}^{2}} .
$$

Since QCD is parity conserving, the matrix element of the operators $\tilde{O}_{1}^{(1) p}$ are the same as the SM operators $O_{1}^{(1) p}$, and no strong phase difference occurs between them. Correspondingly, $\tilde{C}_{1}^{(1) p}$ does not contribute to the direct CP asymmetries (see eq. (3.3)). Due to the different color structure of the operators $\tilde{O}_{2}^{(1) p}$, however, a different strong phase can be expected in their matrix elements and a non-zero contribution to the direct $\mathrm{CP}$ asymmetries can be generated by the weak phase in $\tilde{C}_{2}^{(1) p} \propto X_{c p} X_{u p}^{*}$.

Contributions to $D^{0}-\bar{D}^{0}$ mixing are first induced at the loop level (see diagram (b) in figure 9)

$$
\tilde{C}_{1}^{(2) D} \simeq-\frac{1}{16 \pi^{2}} \frac{N_{c}+3}{32 M_{\phi_{6}}^{2}}\left(\sum_{p=d, s} X_{u p}^{*} X_{c p}\right)^{2},
$$

where, for simplicity, we restrict ourselves to couplings of the diquark to the first two generations of quarks and expand the result in small quark masses.

We note that even with small couplings to quarks $\lesssim 0.1$, sizable effects in flavor observables can be generated. Consequently, dijet searches at hadron colliders do not lead to relevant constraints. Important collider constraints on diquarks, however, come from the

\footnotetext{
${ }^{5}$ All other possibilities are scalars that can also have lepton number violating couplings. One example is a $\mathrm{SU}(3)$ triplet, $\mathrm{SU}(2)_{L}$ singlet with hypercharge $-1 / 3$ which corresponds to a right-handed down squark with $R$-parity violating couplings.
} 


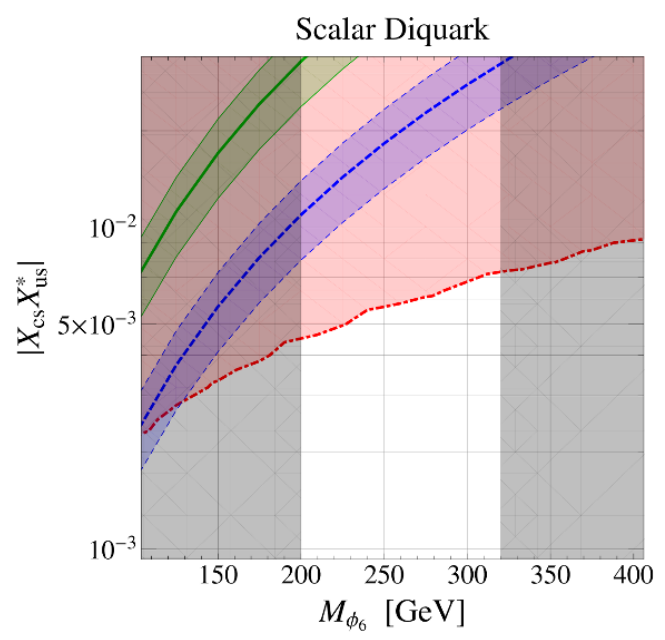

Figure 10. Regions in the $M_{\phi_{6}}-\left|X_{c s} X_{u s}^{*}\right|$ plane compatible with the data on $\Delta A_{\mathrm{CP}}$, wA at the $1 \sigma$ level in the diquark model, setting $\operatorname{Arg}\left(X_{c s} X_{u s}^{*}\right)=\pi / 2$. The green (solid) band corresponds to the expressions for the decay amplitude obtained in naïve factorization, the blue (dashed) band assumes an enhancement of the hadronic matrix elements by a factor of 3. The red (dash-dotted) region is excluded by $D^{0}-\bar{D}^{0}$ constraints. The vertical shaded bands are excluded by dijet pair searches at LHC.

dijet pair searches at LHC. Using MadGraph 5 to simulate diquark pair production, we find that the ATLAS search excludes the region from $100 \mathrm{GeV}$ to $200 \mathrm{GeV}$. The CMS search excludes diquarks with masses from $320 \mathrm{GeV}$ until $1000 \mathrm{GeV}$ assuming an acceptance of $13 \%$.

In figure 10, we show regions in the $M_{\phi_{6}}-\left|X_{c s} X_{u s}^{*}\right|$ plane compatible with the data for $\Delta A_{\mathrm{CP}}$, wA at the $1 \sigma$ level, considering only $X_{c s}$ and $X_{u s}$ to be non-zero and setting $\operatorname{Arg}\left(X_{c s} X_{u s}^{*}\right)=\pi / 2$. The green (solid) band corresponds to the expression for the decay amplitude in naïve factorization. The blue (dashed) band assumes an enhancement of the hadronic matrix elements by a factor of 3 . The red (dash-dotted) region is excluded by the $D^{0}-\bar{D}^{0}$ mixing constraints. The vertical bands are excluded by the dijet pair searches at ATLAS (left band) and CMS (right band). Since only strange quark couplings are considered, $\Delta A_{\mathrm{CP}}$ is entirely generated by $A_{\mathrm{CP}}\left(K^{+} K^{-}\right)$in this setup. Despite the fact that the $D \rightarrow K^{+} K^{-}$decay amplitude arises already at the tree level while $D^{0}-\bar{D}^{0}$ mixing is only induced at the loop level, constraints from $D^{0}-\bar{D}^{0}$ mixing combined with the dijet pair searches exclude a sizable $\Delta A_{\mathrm{CP}}$ unless the hadronic matrix elements are enhanced by a factor more than 3 . This is due to the fact that the contributions to $\Delta A_{\mathrm{CP}}$ coming from the operator $\tilde{O}_{2}^{(1) s}$ are color suppressed and neither enhanced by RG effects nor by chiral factors. Considering only the couplings $X_{c d}$ and $X_{u d}$ leads to an analogous situation, but in this case $\Delta A_{\mathrm{CP}}$ entirely stems from $A_{\mathrm{CP}}\left(\pi^{+} \pi^{-}\right)$.

If all 4 couplings $X_{c s}, X_{u s}, X_{c d}$ and $X_{u d}$ are present, the $D^{0}-\bar{D}^{0}$ mixing constraint can be strongly reduced by assuming a GIM-like mechanism, i.e. assuming the coupling matrix $X$ to be (approximately) unitary. This happens, for example, if $X$ is (approximately) proportional to unity in the weak eigenstate basis for the quarks. In that case, however, $A_{\mathrm{CP}}\left(\pi^{+} \pi^{-}\right) \simeq A_{\mathrm{CP}}\left(K^{+} K^{-}\right)$and the difference between the two, $\Delta A_{\mathrm{CP}}$, is very small. 


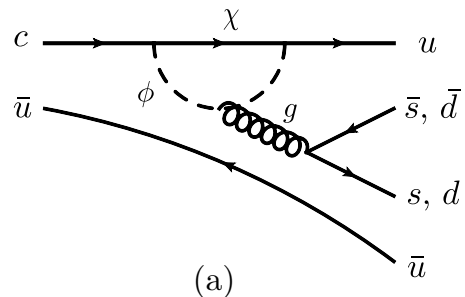

(a)

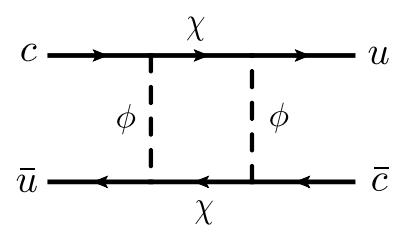

(b)

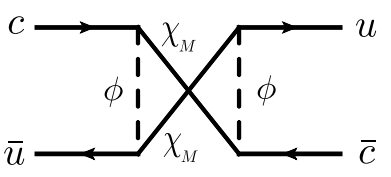

(c)

Figure 11. Example Feynman diagrams that contribute to (a) the $D \rightarrow K^{+} K^{-}$and $D \rightarrow \pi^{+} \pi^{-}$ decay amplitudes and (b and c) $D^{0}-\bar{D}^{0}$ mixing in the discussed models with fermion + scalar loops.

In addition, also loop contributions to kaon mixing are induced if all four couplings are present. Apart from a contribution analogous to eq. (4.31), mixed $\phi_{6}-W$ loops (shown in diagram (c) of figure 9) can become very important. For the latter, we find

$$
\begin{aligned}
C_{4}^{(2) K}=C_{5}^{(2) K}= & \frac{g^{2}}{16 \pi^{2}}\left(V_{c s} V_{c d}^{*}\right)\left(X_{c s} X_{c d}^{*}\right) \\
& \times \frac{1}{2 M_{\phi_{6}}^{2}} \frac{m_{c}^{2}}{M_{W}^{2}} \log \left(\frac{m_{c}^{2}}{M_{\phi_{6}}^{2}}\right),
\end{aligned}
$$

where we expand the result in the charm quark mass and keep only the leading term that is enhanced by a large logarithm. Despite the suppression by $m_{c}^{2} / M_{W}^{2}$, these contributions are very relevant because the matrix elements of $O_{4}^{(2) K}$ and $O_{5}^{(2) K}$ are strongly chirally enhanced and enhanced by renormalization group effects. ${ }^{6}$ Furthermore, the simultaneous presence of $X_{u s}$ and $X_{u d}$ also leads to tree level contributions to $\epsilon^{\prime} / \epsilon$.

Even after tuning phases in order to minimize constraints from CPV in kaon mixing and $\epsilon^{\prime} / \epsilon$, we do not find any region in parameter space of the considered diquark model in which a sizable $\Delta A_{\mathrm{CP}}$ is compatible with all constraints.

\section{New physics contributions to gluon penguins}

We now consider possibilities for new physics in the gluon penguin diagrams at the 1-loop level. We consider models with new fermion and scalar fields, where the fermion is Dirac or Majorana and with or without GIM suppression in section 5.1 and section 5.2, respectively. A scenario with chirally enhanced magnetic penguins is considered in section 5.3. As discussed in the Introduction, the chiral enhancement of the decay amplitudes allows such a scenario to be the least constrained by $D^{0}-\bar{D}^{0}$ mixing.

\subsection{Fermion + scalar loop without GIM mechanism}

We add one heavy fermion $\chi$ and one heavy scalar $\phi$ to the SM that couple with righthanded up type quarks

$$
\mathcal{L}_{\text {int }}=X_{u} \bar{u}_{R} \chi \phi+X_{c} \bar{c}_{R} \chi \phi+X_{t} \bar{t}_{R} \chi \phi+\text { h.c. . }
$$

\footnotetext{
${ }^{6}$ The analogous contribution to $D^{0}-\bar{D}^{0}$ mixing is suppressed by the strange quark mass and has a much smaller chiral enhancement from the matrix elements: it is therefore negligible.
} 
The couplings $X_{i}$ necessarily violate flavor and are free complex parameters. In the following we consider the case where the new particles are $\mathrm{SU}(2)_{L}$ singlets. We assume the fermion is electrically neutral and a $\mathrm{SU}(3)$ singlet and the scalar is a $\mathrm{SU}(3)$ triplet. Different quantum number assignments do not lead to qualitatively different results concerning the $D \rightarrow K^{+} K^{-}$and $D \rightarrow \pi^{+} \pi^{-}$decay amplitudes. We do not consider new fields with flavor breaking couplings to left handed quarks to avoid constraints from kaon mixing and $\epsilon^{\prime} / \epsilon$.

The Lagrangian considered in this framework incorporates an accidental $Z_{2}$ symmetry where $\chi$ and $\phi$ are odd under the symmetry while the SM fields are even. In the considered minimal framework, the lightest of $\chi$ and $\phi$ is therefore stable and becomes the dark matter. If the colored scalar is lighter than the fermion, additional interactions have to be introduced to prevent the scalar from being absolutely stable. If these additional interactions are weak enough such that the scalar remains stable on detector scales, bounds from searches for long-lived particles apply [90, 91]. If instead the scalar decays promptly, then it is highly model dependent whether or not the considered scenario is excluded by current collider searches.

In the case where the neutral fermion is the lighter particle, SUSY searches with jets $+\mathbb{E}_{T}$ at the Tevatron $[92,93]$ and LHC $[94,95]$ provide constraints to the model. In particular, both ATLAS [94] and CMS [95] give bounds on the production cross section for a simplified model that contains the first and second squark generations and a neutralino lightest supersymmetry particle (LSP). We simulate the production cross section using Prospino 2.1 [96] and set all the superpartner masses to be $4.5 \mathrm{TeV}$ except the relevant squarks and the LSP, following [94]. The obtained cross section is then scaled by $1 / 8$ since in our minimal scenario there is only one colored scalar instead of eight. We find that neither the ATLAS nor the CMS searches currently put bounds on our model.

The discussion of the collider constraints is the same regardless of whether the neutral fermion is a Majorana or a Dirac particle. On the other hand, the nature of the fermion plays a crucial role in the low energy phenomenology of the considered model.

\subsubsection{Dirac fermion}

The interactions specified in eq. (5.1) lead to 1-loop gluon penguin contributions to the Wilson coefficients of the $\Delta F=1$ effective Hamiltonian that are shown in diagram (a) of figure 11 and lead to

$$
\begin{aligned}
& \tilde{C}_{6}^{(1)}=\frac{\alpha_{s}}{4 \pi} X_{u} X_{c}^{*} \frac{1}{8 M_{\phi}^{2}} p(z) \\
& \tilde{C}_{3}^{(1)}=\tilde{C}_{5}^{(1)}=-\frac{1}{N_{c}} \tilde{C}_{4}^{(1)}=-\frac{1}{N_{c}} \tilde{C}_{6}^{(1)}, \\
& \tilde{C}_{8 g}^{(1)}=X_{u} X_{c}^{*} \frac{1}{4 M_{\phi}^{2}} g(z) .
\end{aligned}
$$

The loop functions depend on the ratio of the fermion and scalar masses $z=M_{\chi}^{2} / M_{\phi}^{2}$. We find $p(1)=-1 / 24, g(1)=1 / 48$. Their full analytical expressions can be found in appendix C. The relation between the Wilson coefficients $C_{3,4,5,6}^{(1)}$ of the QCD penguin operators is universal for all models where they are induced by gluon penguins. 

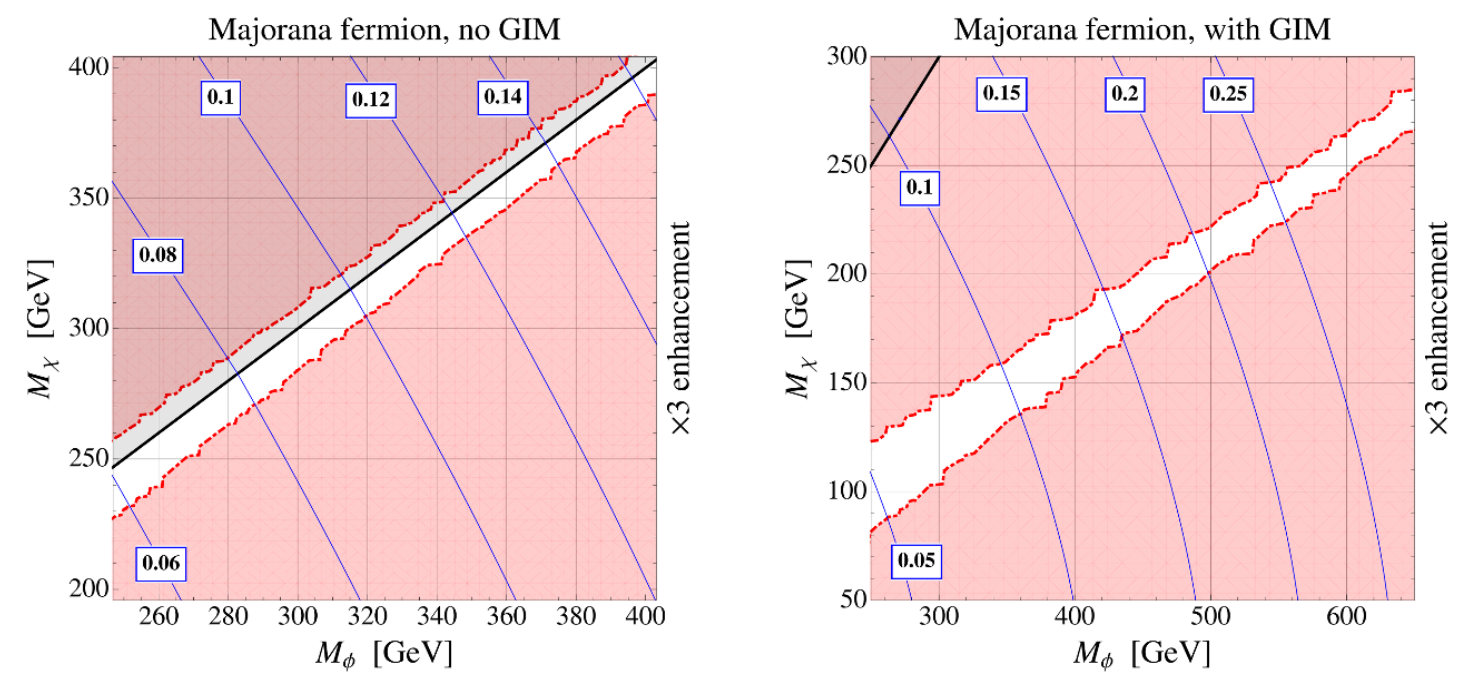

Figure 12. The $M_{\phi}-M_{\chi}$ plane in the models where a gluon penguin is induced by a Majorana fermion - scalar loop without GIM mechanism (left) and with GIM mechanism (right). The blue contours indicate the (left) $\left|X_{u} X_{c}^{*}\right|=0.06, \ldots 0.14$ or (right) $\left|\delta_{c u}\right|=0.05, \ldots 0.25$ values in agreement with the measured $\Delta A_{\mathrm{CP}}$, wA, assuming maximal phases of $\pi / 2$. An enhancement of the hadronic matrix elements by a factor of 3 is assumed in both plots. The red (dash-dotted) region is excluded by the $D^{0}-\bar{D}^{0}$ mixing constraints. In the region above the black (solid diagonal) line, the colored scalar is lighter than the fermion.

The couplings in eq. (5.1) also lead, in principle, to 1-loop box contributions to the $\Delta F=1$ effective Hamiltonian. These contributions, however, are strongly suppressed by an additional factor of $\left|X_{u}\right|^{2}$ and are therefore negligible.

Finally, 1-loop box contributions to $D^{0}-\bar{D}^{0}$ mixing are also induced (see diagram (b) of figure 11)

$$
\tilde{C}_{1}^{(2) D}=\frac{\left(X_{u} X_{c}^{*}\right)^{2}}{16 \pi^{2}} \frac{1}{M_{\phi}^{2}} \frac{1}{8} f(z),
$$

with $f(1)=-1 / 3$. The analytical expression for $f$ is given in appendix C.

We find that $D^{0}-\bar{D}^{0}$ mixing constraints exclude NP contributions to $\Delta A_{\mathrm{CP}}$ as large as the world average even if we allow for an enhancement factor of 3 in the hadronic matrix elements.

\subsubsection{Majorana fermion}

We find qualitatively different results for the Majorana fermion. While the gluon penguin contributions to the decay amplitudes are identical for Majorana and Dirac fermions, the box contributions to $D^{0}-\bar{D}^{0}$ mixing differ. In the case of a Majorana fermion, "crossed" box diagrams also exist (see diagram (c) of figure 11). One finds

$$
\tilde{C}_{1}^{(2) D}=\frac{\left(X_{u} X_{c}^{*}\right)^{2}}{16 \pi^{2}} \frac{1}{M_{\phi}^{2}}\left(\frac{1}{8} f(z)+\frac{1}{4} \tilde{f}(z)\right)
$$

with $\tilde{f}(1)=1 / 6$. The analytical expression for $\tilde{f}$ can be found in appendix C. 
The left plot in figure 12 shows the $M_{\phi}-M_{\chi}$ plane in the considered scenario. The blue contours labeled with $0.06, \ldots 0.14$ show the $\left|X_{u} X_{c}^{*}\right|$ values that are required to be in agreement with the measured $\Delta A_{\mathrm{CP}}$, wA at the $1 \sigma$ level, assuming $\operatorname{Arg}\left(X_{u} X_{c}^{*}\right)=\pi / 2$. An enhancement of the hadronic matrix elements by a factor of 3 is assumed. The red (dash-dotted) region is excluded by the $D^{0}-\bar{D}^{0}$ mixing constraints. In the region above the black (solid diagonal) line shaded in dark gray, the colored scalar is lighter than the fermion and would be a stable colored particle: for this region of parameter space, the model must be extended to be viable.

We observe that if the Majorana and scalar masses are equal, $M_{\phi}=M_{\chi}$, the constraint from $D^{0}-\bar{D}^{0}$ mixing can be avoided. Indeed, for such a ratio of masses, the box and crossed box contributions to the mixing amplitude cancel. The exact mass ratio where such a cancellation occurs depends on the quantum numbers of the scalar and the Majorana fermion. For example, in the well know SUSY case of squark - gluino boxes, the cancellation occurs for $M_{\tilde{g}} \simeq 1.6 \times M_{\tilde{q}}$ (see [97] for a recent discussion.). Because of this cancellation, there exists a narrow region of parameter space where the NP contribution to $\Delta A_{\mathrm{CP}}$ can explain the measured value. The same is in principle true without assuming any enhancement of the hadronic matrix elements. In such a case, however the $D^{0}-\bar{D}^{0}$ mixing constraints hardly allow any deviation from the $M_{\phi}=M_{\chi}$ line.

\subsection{Fermion + scalar loop with GIM mechanism}

We consider now a framework that allows the incorporation of a GIM-like mechanism. In addition to the heavy fermion, we introduce 3 scalars, $\phi_{u, c, t}$, which are scalar partners of the 3 right-handed up-type quarks. If the scalars couple universally to quarks and are approximately degenerate in mass, summing over the three scalar loops strongly suppresses flavor changing neutral current processes. As discussed in [98] for this setup, $\Delta F=2$ processes are more strongly GIM suppressed with respect to $\Delta F=1$ processes and we expect more room for NP in the decay amplitudes.

We consider the following flavor universal couplings between the quarks and scalars

$$
\mathcal{L}_{\text {int }}=g_{\phi} \bar{u}_{R} \chi \phi_{u}+g_{\phi} \bar{c}_{R} \chi \phi_{c}+g_{\phi} \bar{t}_{R} \chi \phi_{t}+\text { h.c. . }
$$

In the basis where the quark masses are diagonal, we write the mass matrix for the scalars as

$$
\hat{M}_{\phi}^{2}=M_{\phi}^{2} \mathbb{I}+M_{\phi}^{2} \delta,
$$

where $\delta_{i j} \ll 1$ are a source of flavor violation. Such a setup is, for example, realized in the MSSM by gluinos and right-handed up squarks. Here, we focus on the case where the new particles are $\mathrm{SU}(2)_{L}$ singlets, the scalars are $\mathrm{SU}(3)$ triplets, and the fermion is a SU(3) singlet. Different quantum number assignments do not lead to qualitatively different results concerning the $D \rightarrow K^{+} K^{-}$and $D \rightarrow \pi^{+} \pi^{-}$decay amplitudes.

Similar to the scenario without the GIM mechanism discussed above, the framework considered here is only mildly constrained by collider searches. The difference with respect to the case without GIM mechanism is that now there are three scalars, and the production cross section for scalar pair production is therefore three times larger than in section 5.1. On 
the other hand, one of the scalars decays predominantly into top quarks and the constraint from squark pair production with direct decays into light quarks and the lightest neutralino from $[94,95]$ cannot be applied for this scalar. Analogously to the previous scenario without GIM, we scale the production cross section obtained by Prospino 2.1 by the ratio of the number of scalars in the model, i.e. a 2/8 scaling. We find that the ATLAS search [94] does not put any bound on our model. The CMS search [95] on the other hand excludes a small corner of parameter space with scalar masses between $300 \mathrm{GeV}$ and $350 \mathrm{GeV}$ and fermion masses below $\simeq 100 \mathrm{GeV}$. This holds regardless of whether the fermion is Majorana or Dirac. The flavor phenomenology is, however, qualitatively different for the two cases.

\subsubsection{Dirac fermion}

The 1-loop gluon penguin contributions to the decay amplitudes are

$$
\begin{aligned}
\tilde{C}_{6}^{(1)} & =\frac{\alpha_{s}}{4 \pi} \delta_{u c} \frac{g_{\phi}^{2}}{8 M_{\phi}^{2}} P(z), \\
\tilde{C}_{3}^{(1)} & =\tilde{C}_{5}^{(1)}=-\frac{1}{N_{c}} \tilde{C}_{4}^{(1)}=-\frac{1}{N_{c}} \tilde{C}_{6}^{(1)}, \\
\tilde{C}_{8 g}^{(1)} & =\delta_{u c} \frac{g_{\phi}^{2}}{4 M_{\phi}^{2}} G(z),
\end{aligned}
$$

where $\delta_{u c}=\delta_{c u}^{*}$ is a complex dimensionless parameter of flavor violation as defined in eq. (5.6). For the loop functions, we find $P(1)=1 / 30, G(1)=-1 / 80$. Their analytical expressions can be found in the appendix.

Now, 1-loop box contributions to the $\Delta F=1$ effective Hamiltonian are not suppressed by additional small mixing angles. For the minimal set of couplings defined in eq. (5.5), we find

$$
\tilde{C}_{3}^{(1)}=\frac{1}{2} \tilde{C}_{9}^{(1)}=\frac{g_{\phi}^{4}}{16 \pi^{2}} \frac{\delta_{u c}}{12 M_{\phi}^{2}} B(z),
$$

where $B(1)=1 / 48$. Its analytical expression can be found in appendix C. We remark that in naïve factorization, this combination of Wilson coefficients does not contribute directly to the $D \rightarrow K^{+} K^{-}$and $D \rightarrow \pi^{+} \pi^{-}$decays that have either strange or down quarks in the final state. ${ }^{7}$ Through renormalization group running, however, the other QCD penguin operators (in particular $\tilde{O}_{6}^{(1)}$ ) are induced and lead to non-zero contributions. Although such contributions are subleading, they are included in our numerical analysis.

Finally, the 1-loop box contributions to $D^{0}-\bar{D}^{0}$ mixing are given by

$$
\tilde{C}_{1}^{(2) D}=\frac{\delta_{u c}^{2}}{16 \pi^{2}} \frac{g_{\phi}^{4}}{M_{\phi}^{2}} \frac{1}{8} F(z)
$$

with $F(1)=-1 / 30$. The analytical expression for $F$ can be found in appendix C.

Similar to the situation without a GIM mechanism, we find that the gluon penguins that are induced by a Dirac fermion loop cannot viably account for the measured $\Delta A_{\mathrm{CP}}$, WA, even if an enhancement of the hadronic matrix elements by a factor of 3 is assumed.

\footnotetext{
${ }^{7}$ They do contribute in QCD factorization via annihilation diagrams that we do not consider here.
} 

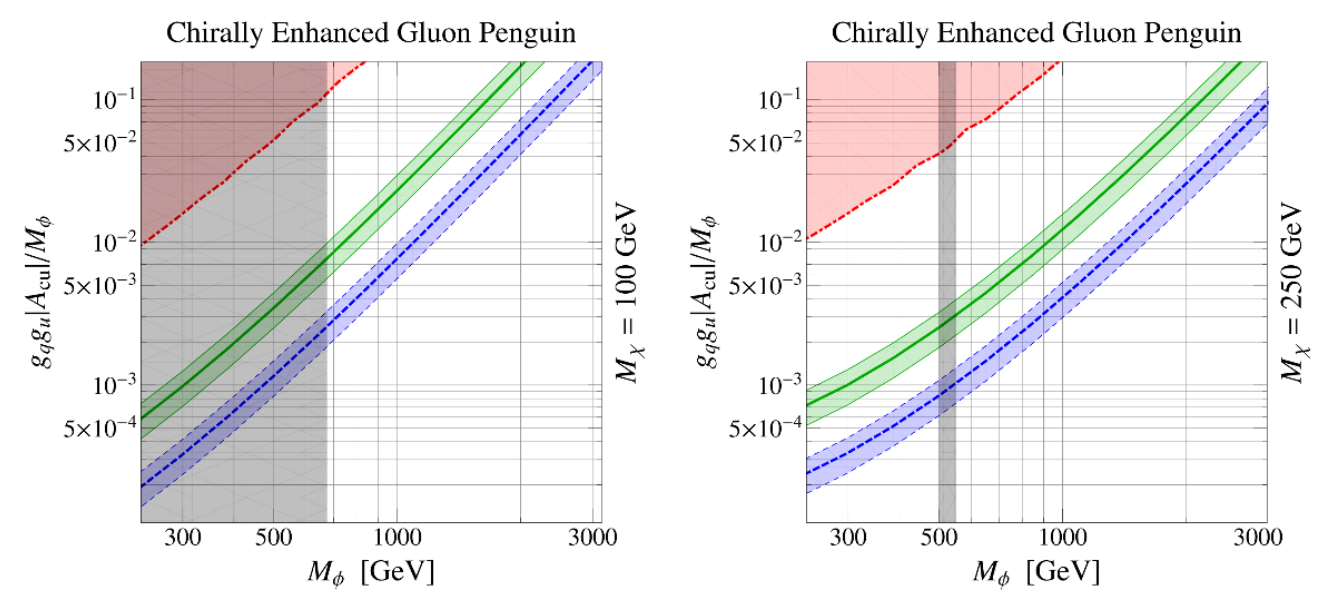

Figure 13. Regions in the $M_{\phi}-g_{u} g_{q}\left|A_{c u}\right| / M_{\phi}$ plane compatible with the data for $\Delta A_{\mathrm{CP}}$, WA at the $1 \sigma$ level in the model with chirally enhanced gluon penguins, setting $\operatorname{Arg}\left(A_{c u}\right)=\pi / 3$. In the left (right) plot $M_{\chi}=100(250) \mathrm{GeV}$. The green (solid) band corresponds to the expression for the decay amplitude in naïve factorization, the blue (dashed) band assumes an enhancement by a factor of 3. The red (dash-dotted) region in the upper left corners is excluded by the $D^{0}-\bar{D}^{0}$ mixing constraint. The dark gray vertical region is excluded by jets $+\mathbb{E}_{T}$ searches at LHC.

\subsubsection{Majorana fermion}

The gluon penguin contributions to the decay amplitudes, that are induced by a Majorana fermion - scalar loop, are identical to the Dirac case, but the box contributions to the decay amplitudes and to $D^{0}-\bar{D}^{0}$ mixing differ. Adding the "crossed" boxes, we find

$$
\begin{aligned}
\tilde{C}_{3}^{(1)}=\frac{1}{2} \tilde{C}_{9}^{(1)} & =\frac{g_{\phi}^{4}}{16 \pi^{2}} \frac{\delta_{u c}}{12 M_{\phi}^{2}}\left(B(z)-\frac{1}{2} \tilde{B}(z)\right), \\
\tilde{C}_{1}^{(2) D} & =\frac{\delta_{u c}^{2}}{16 \pi^{2}} \frac{g_{\phi}^{4}}{M_{\phi}^{2}}\left(\frac{1}{8} F(z)+\frac{1}{4} \tilde{F}(z)\right) .
\end{aligned}
$$

with $\tilde{B}(1)=-1 / 12, \tilde{F}(1)=1 / 20$. Their analytical expressions can be found in appendix C.

The corresponding situation in the $M_{\phi}-M_{\chi}$ plane is shown in the right plot in figure 12, assuming an enhancement of the hadronic matrix elements by a factor of 3 . The blue contours labeled with $0.05, \ldots 0.25$ show the $\left|\delta_{u c}\right|$ that are required for agreement with the measured $\Delta A_{\mathrm{CP}}$, wA at the $1 \sigma$ level, setting $\operatorname{Arg}\left(\delta_{u c}\right)=\pi / 2$. Now, the ratio of the scalar to Majorana mass where the box contributions to meson mixing cancel is $M_{\phi} \simeq 2.3 \times M_{\chi}$. We observe that constraints from $D^{0}-\bar{D}^{0}$ mixing leave more regions of parameter space open, as expected. Still, the measured value for $\Delta A_{\mathrm{CP}}$ can be explained by NP contributions only in a narrow region along the $M_{\phi} \simeq 2.3 \times M_{\chi}$ line.

\subsection{Chirally enhanced magnetic penguins}

Finally, we discuss a setup that leads to chirally enhanced chromomagnetic penguins. We introduce a Majorana fermion $\chi$ that is singlet under the SM gauge group, as well as 
scalar partners, $\phi_{q}$ and $\phi_{u}$, to the left-handed quark doublets $Q$ and the right-handed uptype quarks $U$, respectively. We consider the following interactions among those degrees of freedom

$$
\begin{aligned}
\mathcal{L}_{\text {int, mass }}= & X_{u} \bar{U} \chi \phi_{u}+X_{q} \bar{Q} \chi \phi_{q}+A \phi_{u}^{*} \phi_{q} H+\text { h.c. } \\
& +m_{u}^{2}\left|\phi_{u}\right|^{2}+m_{q}^{2}\left|\phi_{q}\right|^{2}+M_{\chi} \chi \chi .
\end{aligned}
$$

We set the coupling matrices $X_{u}$ and $X_{q}$ to be universal in flavor space: $X_{q}=g_{q} \mathbb{I}, X_{u}=g_{u} \mathbb{I}$. We further assume the mass matrices for the scalars $m_{u}^{2}$ and $m_{q}^{2}$ are universal and, for simplicity, also equal: $m_{q}^{2}=m_{u}^{2}=M_{\phi}^{2} \mathbb{I}$. The only new source of flavor violation is then the trilinear coupling $A$. After electroweak symmetry breaking, the trilinear coupling $A$ leads to mixing between the $\phi_{u}$ and the isospin $+1 / 2$ component of $\phi_{q}$. This setup resembles to a large extent the MSSM with flavor changing trilinear couplings in the up-squark sector as discussed in $[18,26]$.

Both the elements $A_{c u}$ and $A_{u c}$ can lead to $c \rightarrow u$ transitions. Expanding the contribution to the Wilson coefficients in $A_{c u} v / M_{\phi}^{2} \ll 1$, we find

$$
\begin{aligned}
C_{8 g}^{(1)} & =\frac{g_{q} g_{u}}{M_{\phi}^{2}} \frac{v}{m_{c}} \frac{A_{c u} M_{\chi}}{4 M_{\phi}^{2}} \tilde{G}(z), \\
\tilde{C}_{8 g}^{(1)} & =\frac{g_{q} g_{u}}{M_{\phi}^{2}} \frac{v}{m_{c}} \frac{A_{u c}^{*} M_{\chi}}{4 M_{\phi}^{2}} \tilde{G}(z),
\end{aligned}
$$

where $z=M_{\chi}^{2} / M_{\phi}^{2}$ and $\tilde{G}(1)=-1 / 24$. The full analytical expression for the loop function $\tilde{G}$ can be found in appendix C. We highlight the expected chiral enhancement of the magnetic penguins by a factor of $v / m_{c}$.

Box diagrams with the new fermion and the scalars also lead to contributions to $D^{0}-\bar{D}^{0}$ mixing. Considering only the $A_{c u}$ coupling, we find

$$
\tilde{C}_{2}^{(2) D}=\frac{g_{q}^{2} g_{u}^{2}}{16 \pi^{2}} \frac{1}{M_{\phi}^{2}} \frac{\left(A_{c u} v\right)^{2}}{M_{\phi}^{4}} \frac{1}{2} \tilde{F}(z),
$$

where the loop function $\tilde{F}$ is the same as in section 5.2. The $A_{u c}$ coupling leads to the analogous contribution to the coefficient $C_{2}^{(2) D}$. If both couplings are present simultaneously, contributions to $C_{4}^{(2) D}$ and $C_{5}^{(2) D}$ are also generated.

In figure 13, we show regions in the $M_{\phi}-g_{u} g_{q}\left|A_{c u}\right| / M_{\phi}$ plane compatible with the data on $\Delta A_{\mathrm{CP}}$, wA at the $1 \sigma$ level, considering $A_{c u}$ as the only source of flavor violation and setting $\operatorname{Arg}\left(A_{c u}\right)=\pi / 3$. In the plot on the left (right) we set $M_{\chi}=100(250) \mathrm{GeV}$. The green (solid) band corresponds to the expression for the decay amplitude in naïve factorization. The blue (dashed) band assumes an enhancement of the hadronic matrix elements by a factor of 3 . The red (dash dotted) region is excluded by the $D^{0}-\bar{D}^{0}$ mixing constraints. The gray shaded regions are excluded by SUSY searches with jets + $\mathbb{E}_{T}$. Results from ATLAS [94] and CMS [95] indicate that scalar masses up to $675 \mathrm{GeV}$ are excluded for a fermion mass of $100 \mathrm{GeV}$ (see left plot). On the other hand, for a fermion mass of $250 \mathrm{GeV}$, only a small region around $500 \mathrm{GeV}$ is excluded by CMS data alone. For heavier fermion masses, the full range of scalar masses is allowed by present collider constraints. 
Because of the chiral enhancement factor $v / m_{c}$ in the decay amplitudes, $D^{0}-\bar{D}^{0}$ mixing constraints allow for a large $\Delta A_{\mathrm{CP}}$ in the considered setup. Even for scalar masses of $1 \mathrm{TeV}$ and larger, NP contributions to $D^{0}-\bar{D}^{0}$ mixing are more than an order of magnitude below the experimental constraints for $\mathrm{NP}$ contributions to $\Delta A_{\mathrm{CP}}$ that agree with the experimental value.

\section{Conclusions}

The $\mathrm{LHCb}$ measurement of the difference in the time dependent $\mathrm{CP}$ asymmetries in the singly Cabibbo suppressed $D \rightarrow K^{+} K^{-}$and $D \rightarrow \pi^{+} \pi^{-}$decays, $\Delta A_{\mathrm{CP}}$, is the first evidence for charm $\mathrm{CP}$ violation. Although there are large uncertainties in the SM prediction, the measurement could indicate New Physics, and NP interpretations are nevertheless motivated and exciting. In this paper, we studied the effect of NP degrees of freedom for nonstandard direct CPV in the $D \rightarrow K^{+} K^{-}$and $D \rightarrow \pi^{+} \pi^{-}$decays while also considering constraints both from low and high energy experiments.

As is shown in [21], models that give rise to chirally enhanced chromomagnetic $c \rightarrow u$ penguin operators are the least constrained by low energy data and can easily accommodate the large $\Delta A_{\mathrm{CP}}$ value measured by LHCb. The most prominent examples for such models are supersymmetric scenarios as discussed in [18] and very recently in [26]. We studied the chirally enhanced chromomagnetic penguins in the framework of a simplified model that contains scalar partners of the left- and right-handed up-type quarks as well as a Majorana fermion. We confirm that low energy observables, in particular $D^{0}-\bar{D}^{0}$ mixing, as well as collider searches do not significantly constrain the model's parameter space that leads to a sizable $\Delta A_{\mathrm{CP}}$.

Models that contribute to the $D$ meson decays through four fermion operators are generically expected to be strongly constrained by $D^{0}-\bar{D}^{0}$ mixing data [21]. Nonstandard effects in the decays are only possible if the new degrees of freedom mediating the $c \rightarrow u$ transition are very light. In this work, we quantified this statement through a systematic study of models with a minimal set of new degrees of freedom giving rise to four fermion operators both at the tree and the loop level. In summary, we find:

- Flavor changing couplings of the the SM $Z$ boson can induce a $\Delta A_{\mathrm{CP}}$ as large as the observed value if the NP phase is moderately tuned to avoid constraints from indirect $\mathrm{CP}$ violation in $D^{0}-\bar{D}^{0}$ mixing.

- A $Z^{\prime}$ that mediates the $c \rightarrow u$ transition at tree level cannot account for the observed $\Delta A_{\mathrm{CP}}$ due to the combined constraints from $D^{0}-\bar{D}^{0}$ mixing and dijet searches.

- A heavy gluon with a flavor changing tree level $c \rightarrow u$ coupling and with a mass of $200 \mathrm{GeV} \lesssim M_{G^{\prime}} \lesssim 320 \mathrm{GeV}$ cannot fully be excluded as NP explanation for the measured $\Delta A_{\mathrm{CP}}$ if a moderate enhancement of the hadronic matrix elements is allowed.

- In a $2 \mathrm{HDM}$ with $\mathrm{MFV}$, there exist regions of parameter space that can lead to a sizable $\Delta A_{\mathrm{CP}}$. They are characterized by light charged Higgs masses and strongly enhanced couplings of the right handed strange quark to the charged Higgs, with 
respect to its SM Yukawa coupling. Avoiding constraints from perturbativity and $B \rightarrow X_{s} \gamma$, however, requires a considerable amount of fine tuning.

- Scalar octets can also induce large nonstandard effects in a mass window $200 \mathrm{GeV}$ $\lesssim M_{\phi_{8}} \lesssim 320 \mathrm{GeV}$ that is left open by current collider searches. The viable parameter space is analogous to the $2 \mathrm{HDM}$ with MFV model and appears to be, to some extent, fine tuned.

- The scalar diquark model we consider is ruled out by $D^{0}-\bar{D}^{0}$ mixing as a NP explanation of $\Delta A_{\mathrm{CP}}$.

- The minimal models that induce NP effects in the $D$ meson decays through loops with Dirac fermions and scalars are strongly constrained by $D^{0}-\bar{D}^{0}$ mixing data and cannot give rise to a sizable $\Delta A_{\mathrm{CP}}$.

- If Majorana fermions and scalars appear in the loops, then $D^{0}-\bar{D}^{0}$ mixing constraints can be avoided for a particular ratio of Majorana and scalar masses that depends on the exact quantum number assignment for the particles. Correspondingly, in such models there exist regions of parameter space that lead to large $\Delta A_{\mathrm{CP}}$ in agreement with the data.

We note that our results are robust, since changes to the central value of $\Delta A_{\mathrm{CP}}$, new direct search constraints, and the enhancement or suppression of flavor bounds coming from additional field content can be readily applied to our minimal models and our derived Wilson coefficients.

As we showed, the New Physics parameter space favored for an explanation of the $\mathrm{LHCb}$ evidence for charm $\mathrm{CP}$ violation is largely within the current reach of various direct searches at the LHC. Thus the well-known complementarity between low energy flavor measurements and high energy direct probes may prove fruitful as we continue to search for New Physics. Our work emphasizes this synergy by presenting a broad study of minimal New Physics models, discussing both their effects on low energy flavor observables as well as their high energy collider signatures. We have demonstrated that a number of intriguing New Physics models can viably explain the large $\Delta A_{\mathrm{CP}}$ measurement, and we have concretely isolated the interesting parameter spaces of such models which must now be searched directly.

\section{Acknowledgments}

Fermilab is operated by Fermi Research Alliance, LLC under Contract No. De-AC0207CH11359 with the United States Department of Energy. R.P. thanks the National Science Foundation for support under Grants PHY-0757481 and PHY-1068008. R.P. and C.-T.Y are supported by the Fermilab Fellowship in Theoretical Physics. The authors would like to thank T. J. Khoo for useful information regarding the ATLAS jets $+\mathbb{E}_{T}$ search, Andy Cohen and Martin Schmaltz for useful comments, and Daniele Alves, Jonathan Arnold, Bogdan Dobrescu, Stefania Gori and David Straub for useful discussions. 


\section{A Hadronic matrix elements in naïve factorization}

To evaluate the hadronic matrix elements of the operators in the $\Delta F=1$ effective Hamiltonian in eq. (3.1), we use naïve factorization

$$
\begin{aligned}
& \left\langle K^{+} K^{-}\left|\left(\bar{u} \Gamma_{1} s\right)\left(\bar{s} \Gamma_{2} c\right)\right| D^{0}\right\rangle \\
& \simeq\left\langle K^{+}\left|\left(\bar{u} \Gamma_{1} s\right)\right| 0\right\rangle\left\langle K^{-}\left|\left(\bar{s} \Gamma_{2} c\right)\right| D^{0}\right\rangle,
\end{aligned}
$$

where $\Gamma_{i}$ represent the various Dirac and color structures. In this approximation, it is straightforward to evaluate the hadronic matrix elements $\left\langle O_{i}\right\rangle \equiv\left\langle K^{+} K^{-}\left|O_{i}\right| D^{0}\right\rangle$

$$
\begin{aligned}
\left\langle O_{1}^{(1)}\right\rangle & =N_{c}\left\langle O_{2}^{(1)}\right\rangle=N_{c}\left\langle O_{3}^{(1)}\right\rangle=\left\langle O_{4}^{(1)}\right\rangle \\
& =-2 N_{c}\left\langle O_{9}^{(1)}\right\rangle=-2\left\langle O_{10}^{(1)}\right\rangle, \\
\frac{1}{\chi_{f}}\left\langle O_{1}^{(1)}\right\rangle & =N_{c}\left\langle O_{5}^{(1)}\right\rangle=\left\langle O_{6}^{(1)}\right\rangle=-2 N_{c}\left\langle O_{7}^{(1)}\right\rangle \\
& =-2\left\langle O_{8}^{(1)}\right\rangle=-8\left\langle O_{S 1}^{(1)}\right\rangle=-8 N_{c}\left\langle O_{S 2}^{(1)}\right\rangle, \\
\left\langle O_{T 1}^{(1)}\right\rangle & =\left\langle O_{T 2}^{(1)}\right\rangle=0,
\end{aligned}
$$

where $N_{c}=3$ is the number of colors and $\chi_{f}$ is the appropriate chiral factor from eq. (3.4). Using QCD factorization methods, the matrix element of the chromomagnetic operator is $[18]$

$$
\left\langle O_{8 g}^{(1)}\right\rangle=-\frac{\alpha_{s}}{4 \pi} \frac{N_{c}^{2}-1}{N_{c}^{2}}(3+\chi)\left\langle O_{1}^{(1)}\right\rangle .
$$

As QCD conserves parity, the matrix elements of the chirality flipped operators $\tilde{O}_{i}^{(1)}$ are identical to the ones shown above.

\section{B Anomalous dimensions}

For completeness we collect here all the anomalous dimensions of the $\Delta F=1$ operators that are required for our analysis. The LO anomalous dimension matrix that governs the running and mixing of the current-current operators $O_{1,2}^{(1) p}$, the QCD penguin operators $O_{3, \ldots, 6}^{(1)}$ and the QED penguin operators $O_{7, \ldots, 10}^{(1)}$ is given by (see e.g. [99])

$$
\gamma_{1, \ldots, 10}^{0}=\left(\begin{array}{cccccccccc}
\frac{-6}{N_{c}} & 6 & \frac{-2}{3 N_{c}} & \frac{2}{3} & \frac{-2}{3 N_{c}} & \frac{2}{3} & 0 & 0 & 0 & 0 \\
6 & \frac{-6}{N_{c}} & 0 & 0 & 0 & 0 & 0 & 0 & 0 & 0 \\
0 & 0 & \frac{-22}{3 N_{c}} & \frac{22}{3} & \frac{-4}{3 N_{c}} & \frac{4}{3} & 0 & 0 & 0 & 0 \\
0 & 0 & 6-\frac{2 f}{3 N_{c}} & \frac{-6}{N_{c}}+\frac{2 f}{3} & \frac{-2 f}{3 N_{c}} & \frac{2 f}{3} & 0 & 0 & 0 & 0 \\
0 & 0 & 0 & 0 & \frac{6}{N_{c}} & -6 & 0 & 0 & 0 & 0 \\
0 & 0 & \frac{-2 f}{3 N_{c}} & \frac{2 f}{3} & \frac{-2 f}{3 N_{c}} & \frac{6\left(1-N_{c}^{2}\right)}{N_{c}}+\frac{2 f}{3} & 0 & 0 & 0 & 0 \\
0 & 0 & 0 & 0 & 0 & 0 & \frac{6}{N_{c}} & -6 & 0 & 0 \\
0 & 0 & \frac{d-2 u}{3 N_{c}} & \frac{2 u-d}{3} & \frac{d-2 u}{3 N_{c}} & \frac{2 u-d}{3} & 0 & \frac{6\left(1-N_{c}^{2}\right)}{N_{c}} & 0 & 0 \\
0 & 0 & \frac{2}{3 N_{c}} & -\frac{2}{3} & \frac{2}{3 N_{c}} & -\frac{2}{3} & 0 & 0 & \frac{-6}{N_{c}} & 6 \\
0 & 0 & \frac{d-2 u}{3 N_{c}} & \frac{2 u-d}{3} & \frac{d-2 u}{3 N_{c}} & \frac{2 u-d}{3} & 0 & 0 & 6 & \frac{-6}{N_{c}}
\end{array}\right),
$$


where $N_{c}=3$ is the number of colors, $f$ is the number of active quark flavors, and $u$ and $d$ are the numbers of active up- and down-type quarks, respectively.

Leading order running of the chromomagnetic operator $O_{8 g}^{(1)}$ is given by

$$
\gamma_{8 g}^{0}=\frac{4 N_{c}^{2}-8}{N_{c}} .
$$

For the LO anomalous dimension matrix responsible for the running and mixing of the scalar and tensor operators $O_{S 1, S 2}^{(1)}$ and $O_{T 1, T 2}^{(1)}$, we find

$$
\gamma_{S T}^{0}=\left(\begin{array}{cccc}
\frac{6-6 N_{c}^{2}}{N_{c}} & 0 & \frac{1}{N_{c}} & -1 \\
-6 & \frac{6}{N_{c}} & -\frac{1}{2} & \frac{2-N_{c}^{2}}{2 N_{c}} \\
\frac{48}{N_{c}} & -48 & \frac{2 N_{c}^{2}-2}{N_{c}} & 0 \\
-24 & \frac{48-24 N_{c}^{2}}{N_{c}} & 6 & \frac{4 N_{c}^{2}+2}{-N_{c}}
\end{array}\right),
$$

which agrees with [31] once the different conventions for the operators and the $\sigma_{\mu \nu}$ matrix are taken into account. At leading order and in the limit of massless down and strange quarks, the scalar and tensor operators do not mix into other operators.

As QCD conserves parity, the anomalous dimensions for the chirality flipped operators $\tilde{O}_{i}^{(1)}$ are identical to the ones shown above.

\section{Loop functions}

The loop functions $h_{1}$ and $h_{2}$ appear in the charged Higgs contributions to kaon mixing in the $2 \mathrm{HDM}$ in section 4.5

$$
\begin{aligned}
h_{1}(x)= & \frac{1+x}{2(1-x)^{2}}+\frac{x}{(1-x)^{3}} \log (x), \\
h_{2}(x, y)= & \frac{x-4 y}{(1-x)(y-x)}+\frac{3 y^{2} \log (y)}{(1-y)(x-y)^{2}} \\
& +\frac{2 x y-4 y^{2}+x^{2}(3 y-1)}{(1-x)^{2}(y-x)^{2}} \log (x) .
\end{aligned}
$$

The loop functions $p, g, f$ and $\tilde{f}$ appear in the discussion of the model with fermion scalar loops without a GIM mechanism in section 5.1. The functions $p$ and $g$ occur in the expressions for the gluon penguin contributions to the $D$ meson decay amplitudes. The function $f$ comes from the evaluation of a box diagram contributing to $D^{0}-\bar{D}^{0}$ mixing and $\tilde{f}$ comes from the corresponding crossed box diagram

$$
\begin{aligned}
& p(z)=-\frac{2-7 z+11 z^{2}}{36(1-z)^{3}}-\frac{z^{3}}{6(1-z)^{4}} \log (z) \\
& g(z)=\frac{1-5 z-2 z^{2}}{24(1-z)^{3}}-\frac{z^{2}}{4(1-z)^{4}} \log (z) \\
& f(z)=-\frac{1+z}{(1-z)^{2}}-\frac{2 z}{(1-z)^{3}} \log (z) \\
& \tilde{f}(z)=-\frac{2 z}{(1-z)^{2}}-\frac{z(1+z)}{(1-z)^{3}} \log (z)
\end{aligned}
$$


The loop functions $P, G, F$ and $\tilde{F}$ are the analogues to $p, g, f$ and $\tilde{f}$ in the fermion - scalar model with a GIM mechanism discussed in section 5.2. The functions $B$ and $\tilde{B}$ appear in the box and crossed box contributions to the $D$ meson decay amplitudes of that framework:

$$
\begin{aligned}
& P(z)=\frac{1-5 z+13 z^{2}+3 z^{3}}{18(1-z)^{4}}+\frac{2 z^{3}}{3(1-z)^{5}} \log (z), \\
& G(z)=\frac{-1+8 z+17 z^{2}}{24(1-z)^{4}}+\frac{z^{2}(3+z)}{4(1-z)^{5}} \log (z) \\
& B(z)=\frac{1+5 z}{8(1-z)^{3}}+\frac{z(2+z)}{4(1-z)^{4}} \log (z) \\
& \tilde{B}(z)=\frac{z(5+z)}{2(1-z)^{3}}+\frac{z(1+2 z)}{(1-z)^{4}} \log (z) \\
& F(z)=-\frac{1+10 z+z^{2}}{3(1-z)^{4}}-\frac{2 z(1+z)}{(1-z)^{5}} \log (z), \\
& \tilde{F}(z)=-\frac{z\left(17+8 z-z^{2}\right)}{6(1-z)^{4}}-\frac{z(1+3 z)}{(1-z)^{5}} \log (z) .
\end{aligned}
$$

Finally, the loop function $\tilde{G}$ appears in the expression for the chromomagnetic penguin loop in section 5.3

$$
\tilde{G}(z)=-\frac{1+5 z}{4(1-z)^{3}}-\frac{z(2+z)}{2(1-z)^{4}} \log (z)
$$

Open Access. This article is distributed under the terms of the Creative Commons Attribution License which permits any use, distribution and reproduction in any medium, provided the original author(s) and source are credited.

\section{References}

[1] LHCB collaboration, R. Aaij et al., A search for time-integrated CP-violation in $D^{0} \rightarrow h^{-} h^{+}$decays, LHCb-CONF-2011-061 [arXiv:1112.0938].

[2] CDF collaboration, T. Aaltonen et al., Measurement of CP-violating asymmetries in $D^{0} \rightarrow \pi^{+} \pi^{-}$and $D^{0} \rightarrow K^{-} K^{+}$decays at CDF, Phys. Rev. D 85 (2012) 012009 [arXiv:1111.5023] [INSPIRE].

[3] Heavy Flavor Averaging Group collaboration, D. Asner et al., Averages of b-hadron, c-hadron and $\tau$-lepton properties, arXiv:1010.1589 [Online update at ] [INSPIRE].

[4] BABAR collaboration, B. Aubert et al., Search for CP-violation in the decays $D^{0} \rightarrow K^{-} K^{+}$ and $D^{0} \rightarrow \pi^{0} \pi^{+}$, Phys. Rev. Lett. 100 (2008) 061803 [arXiv:0709.2715] [InSPIRE].

[5] BeLle collaboration, M. Staric et al., Measurement of CP asymmetry in Cabibbo suppressed $D^{0}$ decays, Phys. Lett. B 670 (2008) 190 [arXiv:0807.0148] [INSPIRE].

[6] J. Brod, A.L. Kagan and J. Zupan, On the size of direct CP-violation in singly Cabibbo-suppressed D decays, arXiv:1111.5000 [INSPIRE]. 
[7] H.-Y. Cheng and C.-W. Chiang, Direct CP-violation in two-body hadronic charmed meson decays, Phys. Rev. D 85 (2012) 034036 [arXiv:1201.0785] [inSPIRE].

[8] D. Pirtskhalava and P. Uttayarat, $C P$ violation and flavor $S U(3)$ breaking in d-meson decays, arXiv: 1112.5451 [INSPIRE].

[9] B. Bhattacharya, M. Gronau and J.L. Rosner, CP asymmetries in singly-Cabibbo-suppressed $D$ decays to two pseudoscalar mesons, arXiv:1201.2351 [INSPIRE].

[10] Particle Data Group collaboration, K. Nakamura et al., Review of particle physics, J. Phys. G 37 (2010) 075021 [inSPIRE].

[11] G. Blaylock, A. Seiden and Y. Nir, The role of CP-violation in $D^{0}-\bar{D}^{0}$ mixing, Phys. Lett. B 355 (1995) 555 [hep-ph/9504306] [INSPIRE].

[12] S. Bianco, F. Fabbri, D. Benson and I. Bigi, A Cicerone for the physics of charm, Riv. Nuovo Cim. 26N7 (2003) 1 [hep-ex/0309021] [INSPIRE].

[13] E. Golowich, J. Hewett, S. Pakvasa and A.A. Petrov, Implications of $D^{0}-\bar{D}^{0}$ mixing for new physics, Phys. Rev. D 76 (2007) 095009 [arXiv: 0705.3650] [InSPIRE].

[14] O. Gedalia, Y. Grossman, Y. Nir and G. Perez, Lessons from recent measurements of $D^{0}-\bar{D}^{0}$ mixing, Phys. Rev. D 80 (2009) 055024 [arXiv:0906.1879] [INSPIRE].

[15] I.I. Bigi, M. Blanke, A.J. Buras and S. Recksiegel, $C P$ violation in $D^{0}-\bar{D}^{0}$ oscillations: general considerations and applications to the littlest Higgs model with T-Parity, JHEP 07 (2009) 097 [arXiv: 0904.1545] [INSPIRE].

[16] A.L. Kagan and M.D. Sokoloff, On indirect CP-violation and implications for $D^{0}-\bar{D}^{0}$ and $B_{s}-\bar{B}_{s}$ mixing, Phys. Rev. D 80 (2009) 076008 [arXiv:0907.3917] [InSPIRE].

[17] W. Altmannshofer, A.J. Buras and P. Paradisi, A lower bound on hadronic EDMs from CP-violation in $D^{0}-\bar{D}^{0}$ mixing in SUSY alignment models, Phys. Lett. B 688 (2010) 202 [arXiv: 1001.3835] [INSPIRE].

[18] Y. Grossman, A.L. Kagan and Y. Nir, New physics and CP-violation in singly Cabibbo suppressed D decays, Phys. Rev. D 75 (2007) 036008 [hep-ph/0609178] [INSPIRE].

[19] I.I. Bigi, A. Paul and S. Recksiegel, Conclusions from CDF results on CP-violation in $D^{0} \rightarrow \pi^{+} \pi^{-} K^{+} K^{-}$and future tasks, JHEP 06 (2011) 089 [arXiv:1103.5785] [INSPIRE].

[20] I.I. Bigi and A. Paul, On CP asymmetries in two-, three- and four-body D decays, JHEP 03 (2012) 021 [arXiv:1110.2862] [InSPIRE].

[21] G. Isidori, J.F. Kamenik, Z. Ligeti and G. Perez, Implications of the LHCb evidence for charm CP-violation, arXiv:1111.4987 [INSPIRE].

[22] K. Wang and G. Zhu, Can up FCNC solve the $\Delta A_{\mathrm{CP}}$ puzzle?, Phys. Lett. B 709 (2012) 362 [arXiv:1111.5196] [INSPIRE].

[23] A. Rozanov and M. Vysotsky, $\left(\Delta A_{\mathrm{CP}}\right)_{\mathrm{LHCb}}$ and the fourth generation, arXiv:1111.6949 [INSPIRE].

[24] Y. Hochberg and Y. Nir, Relating direct CP-violation in D decays and the forward-backward asymmetry in $t \bar{t}$ production, arXiv:1112.5268 [INSPIRE].

[25] X. Chang, M.-K. Du, C. Liu, J.-S. Lu and S. Yang, LHCb $\Delta A_{\mathrm{CP}}$ of D meson and R-parity violation, arXiv:1201.2565 [INSPIRE]. 
[26] G.F. Giudice, G. Isidori and P. Paradisi, Direct CP-violation in charm and flavor mixing beyond the SM, arXiv:1201.6204 [INSPIRE].

[27] M. Golden and B. Grinstein, Enhanced CP-violations in hadronic charm decays, Phys. Lett. B 222 (1989) 501 [INSPIRE].

[28] M. Beneke, G. Buchalla, M. Neubert and C.T. Sachrajda, QCD factorization for $B \rightarrow \pi \pi$ decays: strong phases and CP-violation in the heavy quark limit, Phys. Rev. Lett. 83 (1999) 1914 [hep-ph/9905312] [INSPIRE].

[29] M. Beneke, G. Buchalla, M. Neubert and C.T. Sachrajda, QCD factorization for exclusive, nonleptonic $B$ meson decays: general arguments and the case of heavy light final states, Nucl. Phys. B 591 (2000) 313 [hep-ph/0006124] [INSPIRE].

[30] M. Ciuchini, E. Franco, V. Lubicz, G. Martinelli, I. Scimemi, et al., Next-to-leading order QCD corrections to delta $F=2$ effective Hamiltonians, Nucl. Phys. B 523 (1998) 501 [hep-ph/9711402] [INSPIRE].

[31] A.J. Buras, M. Misiak and J. Urban, Two loop QCD anomalous dimensions of flavor changing four quark operators within and beyond the standard model, Nucl. Phys. B 586 (2000) 397 [hep-ph/0005183] [INSPIRE].

[32] D. Becirevic, V. Giménez, G. Martinelli, M. Papinutto and J. Reyes, $B$ parameters of the complete set of matrix elements of delta $B=2$ operators from the lattice, JHEP 04 (2002) 025 [hep-lat/0110091] [INSPIRE].

[33] R. Babich, N. Garron, C. Hölbling, J. Howard, L. Lellouch, et al., K0 - $\overline{0}$ mixing beyond the standard model and CP-violating electroweak penguins in quenched QCD with exact chiral symmetry, Phys. Rev. D 74 (2006) 073009 [hep-lat/0605016] [INSPIRE].

[34] UTFIT collaboration, M. Bona et al., Model-independent constraints on $\Delta F=2$ operators and the scale of new physics, JHEP 03 (2008) 049 [arXiv:0707.0636] [INSPIRE].

[35] A.F. Falk, Y. Grossman, Z. Ligeti and A.A. Petrov, SU(3) breaking and $D^{0}-\bar{D}^{0}$ mixing, Phys. Rev. D 65 (2002) 054034 [hep-ph/0110317] [INSPIRE].

[36] A.F. Falk, Y. Grossman, Z. Ligeti, Y. Nir and A.A. Petrov, The $D^{0}-\bar{D}^{0}$ mass difference from a dispersion relation, Phys. Rev. D 69 (2004) 114021 [hep-ph/0402204] [INSPIRE].

[37] M. Ciuchini, E. Franco, D. Guadagnoli, V. Lubicz, M. Pierini, et al., D - $\bar{D}$ mixing and new physics: general considerations and constraints on the MSSM, Phys. Lett. B 655 (2007) 162 [hep-ph/0703204] [INSPIRE].

[38] G. Buchalla, G. Hiller and G. Isidori, Phenomenology of nonstandard $Z$ couplings in exclusive semileptonic $b \rightarrow s$ transitions, Phys. Rev. D 63 (2000) 014015 [hep-ph/0006136] [INSPIRE].

[39] Y. Nir and D.J. Silverman, Z mediated flavor changing neutral currents and their implications for CP asymmetries in $B^{0}$ decays, Phys. Rev. D 42 (1990) 1477 [INSPIRE].

[40] P. Langacker and M. Plümacher, Flavor changing effects in theories with a heavy $Z^{\prime}$ boson with family nonuniversal couplings, Phys. Rev. D 62 (2000) 013006 [hep-ph/0001204] [INSPIRE].

[41] P.J. Fox, J. Liu, D. Tucker-Smith and N. Weiner, An effective Z', Phys. Rev. D 84 (2011) 115006 [arXiv: 1104.4127] [INSPIRE]. 
[42] A. Arhrib, K. Cheung, C.-W. Chiang and T.-C. Yuan, Single top-quark production in flavor-changing Z' models, Phys. Rev. D 73 (2006) 075015 [hep-ph/0602175] [INSPIRE].

[43] UA2 collaboration, J. Alitti et al., A search for new intermediate vector mesons and excited quarks decaying to two jets at the CERN pp collider, Nucl. Phys. B 400 (1993) 3 [INSPIRE].

[44] CDF collaboration, F. Abe et al., Search for new particles decaying to dijets at CDF, Phys. Rev. D 55 (1997) 5263 [hep-ex/9702004] [INSPIRE].

[45] CMS collaboration, S. Chatrchyan et al., Search for resonances in the dijet mass spectrum from 7 TeV pp collisions at CMS, Phys. Lett. B 704 (2011) 123 [arXiv:1107.4771] [INSPIRE].

[46] D0 collaboration, V. Abazov et al., Search for new particles in the two jet decay channel with the D0 detector, Phys. Rev. D 69 (2004) 111101 [hep-ex/0308033] [INSPIRE].

[47] ATLAS collaboration, G. Aad et al., Search for new physics in the dijet mass distribution using $1 \mathrm{fb}^{-1}$ of pp collision data at $\sqrt{\mathrm{s}}=7 \mathrm{TeV}$ collected by the ATLAS detector, Phys. Lett. B 708 (2012) 37 [arXiv: 1108.6311] [INSPIRE].

[48] J. Alwall, M. Herquet, F. Maltoni, O. Mattelaer and T. Stelzer, MadGraph 5 : going beyond, JHEP 06 (2011) 128 [arXiv: 1106. 0522] [INSPIRE].

[49] A. Pukhov, E. Boos, M. Dubinin, V. Edneral, V. Ilyin, et al., CompHEP: a package for evaluation of Feynman diagrams and integration over multiparticle phase space, hep-ph/9908288 [inSPIRE].

[50] P. Langacker, The physics of heavy Z' gauge bosons, Rev. Mod. Phys. 81 (2009) 1199 [arXiv: 0801.1345] [INSPIRE].

[51] K. Agashe, G. Perez and A. Soni, Flavor structure of warped extra dimension models, Phys. Rev. D 71 (2005) 016002 [hep-ph/0408134] [INSPIRE].

[52] U. Haisch and S. Westhoff, Massive color-octet bosons: bounds on effects in top-quark pair production, JHEP 08 (2011) 088 [arXiv:1106.0529] [INSPIRE].

[53] B.A. Dobrescu, K. Kong and R. Mahbubani, Massive color-octet bosons and pairs of resonances at hadron colliders, Phys. Lett. B 670 (2008) 119 [arXiv:0709.2378] [INSPIRE].

[54] ATLAS collaboration, G. Aad et al., Search for massive colored scalars in four-jet final states in $\sqrt{s}=7 \mathrm{TeV}$ proton-proton collisions with the ATLAS detector, Eur. Phys. J. C 71 (2011) 1828 [arXiv:1110.2693] [INSPIRE].

[55] CMS collaboration, Search for new physics in the paired dijet mass spectrum, CMS-EXO-11-016.

[56] M. Schmaltz and C. Spethmann, Two simple $W^{\prime}$ models for the early LHC, JHEP 07 (2011) 046 [arXiv: 1011.5918] [InSPIRE].

[57] G. Branco, P. Ferreira, L. Lavoura, M. Rebelo, M. Sher, et al., Theory and phenomenology of two-Higgs-doublet models, arXiv:1106.0034 [INSPIRE].

[58] G. D'Ambrosio, G. Giudice, G. Isidori and A. Strumia, Minimal flavor violation: an effective field theory approach, Nucl. Phys. B 645 (2002) 155 [hep-ph/0207036] [INSPIRE].

[59] A.J. Buras, M.V. Carlucci, S. Gori and G. Isidori, Higgs-mediated FCNCs: natural flavour conservation vs. Minimal flavour violation, JHEP 10 (2010) 009 [arXiv:1005.5310] [INSPIRE]. 
[60] S. Davidson and H.E. Haber, Basis-independent methods for the two-Higgs-doublet model, Phys. Rev. D 72 (2005) 035004 [Erratum ibid. D 72 (2005) 099902] [hep-ph/0504050] [INSPIRE].

[61] A.J. Buras, G. Isidori and P. Paradisi, EDMs versus $C P V$ in $B_{s, d}$ mixing in two Higgs doublet models with MFV, Phys. Lett. B 694 (2011) 402 [arXiv:1007.5291] [INSPIRE].

[62] M. Trott and M.B. Wise, On theories of enhanced CP-violation in $B_{s, d}$ meson mixing, JHEP 11 (2010) 157 [arXiv:1009.2813] [INSPIRE].

[63] M. Jung, A. Pich and P. Tuzon, Charged-Higgs phenomenology in the aligned two-Higgs-doublet model, JHEP 11 (2010) 003 [arXiv: 1006.0470] [INSPIRE].

[64] J.M. Cline, K. Kainulainen and M. Trott, Electroweak baryogenesis in two Higgs doublet models and B meson anomalies, JHEP 11 (2011) 089 [arXiv:1107.3559] [INSPIRE].

[65] ALEPH collaboration, A. Heister et al., Search for charged Higgs bosons in $e^{+} e^{-}$collisions at energies up to $\sqrt{s}=209 \mathrm{GeV}$, Phys. Lett. B 543 (2002) 1 [hep-ex/0207054] [INSPIRE].

[66] Belle collaboration, K. Ikado et al., Evidence of the purely leptonic decay $B^{-} \rightarrow \tau^{-} \bar{\nu}_{\tau}$, Phys. Rev. Lett. 97 (2006) 251802 [hep-ex/0604018] [INSPIRE].

[67] Belle collaboration, K. Hara et al., Evidence for $B^{-} \rightarrow \tau^{-} \overline{n u}$ with a semileptonic tagging method, Phys. Rev. D 82 (2010) 071101 [arXiv: 1006.4201] [INSPIRE].

[68] BABAR collaboration, B. Aubert et al., A search for $B^{+} \rightarrow l+\nu_{l}$ recoiling against $B^{-} \rightarrow D^{0} l^{-} \bar{\nu} \times$, Phys. Rev. D 81 (2010) 051101 [arXiv:0809.4027] [INSPIRE].

[69] BABAR collaboration, P. del Amo Sanchez et al., Evidence for $B^{+} \rightarrow \tau^{+} \nu_{\tau}$ decays using hadronic B tags, arXiv:1008.0104 [INSPIRE].

[70] Fermilab Lattice and MilC Collaborations collaboration, A. Bazavov et al., $B^{-}$ and d-meson decay constants from three-flavor lattice QCD, arXiv:1112.3051 [INSPIRE].

[71] M. Antonelli, V. Cirigliano, G. Isidori, F. Mescia, M. Moulson, et al., An evaluation of $\left|V_{\mathrm{us}}\right|$ and precise tests of the standard model from world data on leptonic and semileptonic kaon decays, Eur. Phys. J. C 69 (2010) 399 [arXiv:1005.2323] [INSPIRE].

[72] M. Misiak, H. Asatrian, K. Bieri, M. Czakon, A. Czarnecki, et al., Estimate of $B\left(\bar{B} \rightarrow X_{s} \gamma\right)$ at o $\left(\alpha_{s}^{2}\right)$, Phys. Rev. Lett. 98 (2007) 022002 [hep-ph/0609232] [INSPIRE].

[73] W. Altmannshofer, P. Paradisi and D.M. Straub, Model-Independent constraints on new physics in $B \rightarrow S$ transitions, arXiv:1111.1257 [INSPIRE].

[74] ATLAS collaboration, Search for charged Higgs bosons in the $\tau+$ jets final state in $t \bar{t}$ decays with $1.03 \mathrm{fb}^{-1}$ of pp collision data recorded at $\sqrt{s}=7 \mathrm{TeV}$ with the ATLAS experiment, ATLAS-CONF-2011-138 (2011).

[75] CDF collaboration, T. Aaltonen et al., Search for charged Higgs bosons in decays of top quarks in pp collisions at $\sqrt{s}=1.96$ TeV, Phys. Rev. Lett. 103 (2009) 101803 [arXiv: 0907.1269] [INSPIRE].

[76] D0 collaboration, V. Abazov et al., Search for charged Higgs bosons in top quark decays, Phys. Lett. B 682 (2009) 278 [arXiv:0908.1811] [INSPIRE].

[77] ATLAS collaboration, Search for neutral MSSM Higgs bosons decaying to tau ${ }^{+} \tau^{-}$pairs in proton-proton collisions at $\sqrt{s}=7 \mathrm{TeV}$ with the ATLAS detector, ATLAS-CONF-2011-132 (2011). 
[78] D0 collaboration, V.M. Abazov et al., Search for neutral Higgs bosons in the multi-b-jet topology in 5.2fb ${ }^{-1}$ of p $\bar{p}$ collisions at $\sqrt{s}=1.96 \mathrm{TeV}$, Phys. Lett. B 698 (2011) 97 [arXiv: 1011.1931] [INSPIRE].

[79] CDF collaboration, Search for Higgs bosons produced in association with b-quarks, CDF Note 10414, http://www-cdf.fnal.gov/physics/new/hdg/results/3b_susyhiggs_june11/.

[80] B.A. Dobrescu and P.J. Fox, Uplifted supersymmetric Higgs region, Eur. Phys. J. C 70 (2010) 263 [arXiv: 1001.3147] [InSPIRE].

[81] W. Altmannshofer and D.M. Straub, Viability of MSSM scenarios at very large $\tan \beta$, JHEP 09 (2010) 078 [arXiv: 1004.1993] [INSPIRE].

[82] A.V. Manohar and M.B. Wise, Flavor changing neutral currents, an extended scalar sector and the Higgs production rate at the CERN LHC, Phys. Rev. D 74 (2006) 035009 [hep-ph/0606172] [INSPIRE].

[83] C. Burgess, M. Trott and S. Zuberi, Light octet scalars, a heavy Higgs and minimal flavour violation, JHEP 09 (2009) 082 [arXiv: 0907.2696] [INSPIRE].

[84] M.I. Gresham and M.B. Wise, Color octet scalar production at the LHC, Phys. Rev. D 76 (2007) 075003 [arXiv:0706.0909] [INSPIRE].

[85] Y. Bai and B.A. Dobrescu, Heavy octets and Tevatron signals with three or four B jets, JHEP 07 (2011) 100 [arXiv:1012.5814] [InSPIRE].

[86] CDF collaboration, Search for Higgs bosons produced in association with b-quarks, CDF Note 8954, www-cdf .fnal.gov/ twright/higgs3b/cdf8954_higgs3b_v10.pdf.

[87] M. Gerbush, T.J. Khoo, D.J. Phalen, A. Pierce and D. Tucker-Smith, Color-octet scalars at the CERN LHC, Phys. Rev. D 77 (2008) 095003 [arXiv:0710.3133] [INSPIRE].

[88] J.M. Arnold, M. Pospelov, M. Trott and M.B. Wise, Scalar representations and minimal flavor violation, JHEP 01 (2010) 073 [arXiv:0911.2225] [INSPIRE].

[89] G.F. Giudice, B. Gripaios and R. Sundrum, Flavourful production at hadron colliders, JHEP 08 (2011) 055 [arXiv: 1105.3161] [InSPIRE].

[90] ATLAS collaboration, G. Aad et al., Search for massive long-lived highly ionising particles with the ATLAS detector at the LHC, Phys. Lett. B 698 (2011) 353 [arXiv:1102.0459] [INSPIRE].

[91] CMS collaboration, Search for heavy stable charged particles, CMS-EXO-11-022.

[92] D0 collaboration, V. Abazov et al., Search for squarks and gluinos in events with jets and missing transverse energy using $2.1 \mathrm{fb}^{-1}$ of $p \bar{p}$ collision data at $\sqrt{s}=1.96 \mathrm{TeV}$, Phys. Lett. B 660 (2008) 449 [arXiv:0712.3805] [INSPIRE].

[93] CDF collaboration, T. Aaltonen et al., Inclusive search for squark and gluino production in $p \bar{p}$ collisions at $\sqrt{s}=1.96$ TeV, Phys. Rev. Lett. 102 (2009) 121801 [arXiv:0811.2512] [INSPIRE].

[94] ATLAS collaboration, Search for supersymmetry with jets and missing transverse momentum: additional model interpretations, ATLAS-CONF-2011-155 (2011).

[95] CMS collaboration, S. Chatrchyan et al., Search for supersymmetry at the LHC in events with jets and missing transverse energy, Phys. Rev. Lett. 107 (2011) 221804 [arXiv: 1109.2352] [INSPIRE]. 
[96] W. Beenakker, R. Hopker and M. Spira, PROSPINO: a program for the production of supersymmetric particles in next-to-leading order $Q C D$, hep-ph/9611232 [INSPIRE].

[97] A. Crivellin and M. Davidkov, Do squarks have to be degenerate? constraining the mass splitting with kaon and D mixing, Phys. Rev. D 81 (2010) 095004 [arXiv:1002.2653] [INSPIRE].

[98] G.F. Giudice, M. Nardecchia and A. Romanino, Hierarchical soft terms and flavor physics, Nucl. Phys. B 813 (2009) 156 [arXiv:0812.3610] [inSPIRE].

[99] G. Buchalla, A.J. Buras and M.E. Lautenbacher, Weak decays beyond leading logarithms, Rev. Mod. Phys. 68 (1996) 1125 [hep-ph/9512380] [INSPIRE]. 